ESAIM: M2AN 47 (2013) 1017-1035

DOI: $10.1051 / \mathrm{m} 2 \mathrm{an} / 2012055$
ESAIM: Mathematical Modelling and Numerical Analysis

www.esaim-m2an.org

\title{
A COMPARISON OF COUPLED AND UNCOUPLED SOLVERS FOR THE CARDIAC BIDOMAIN MODEL *,**
}

\author{
P. Colli Franzone ${ }^{1}$, L.F. Pavarino ${ }^{2}$ And S. Scacchi ${ }^{2}$
}

\begin{abstract}
The aim of this work is to compare a new uncoupled solver for the cardiac Bidomain model with a usual coupled solver. The Bidomain model describes the bioelectric activity of the cardiac tissue and consists of a system of a non-linear parabolic reaction-diffusion partial differential equation (PDE) and an elliptic linear PDE. This system models at macroscopic level the evolution of the transmembrane and extracellular electric potentials of the anisotropic cardiac tissue. The evolution equation is coupled through the non-linear reaction term with a stiff system of ordinary differential equations (ODEs), the so-called membrane model, describing the ionic currents through the cellular membrane. A novel uncoupled solver for the Bidomain system is here introduced, based on solving twice the parabolic PDE and once the elliptic PDE at each time step, and it is compared with a usual coupled solver. Three-dimensional numerical tests have been performed in order to show that the proposed uncoupled method has the same accuracy of the coupled strategy. Parallel numerical tests on structured meshes have also shown that the uncoupled technique is as scalable as the coupled one. Moreover, the conjugate gradient method preconditioned by Multilevel Hybrid Schwarz preconditioners converges faster for the linear systems deriving from the uncoupled method than from the coupled one. Finally, in all parallel numerical tests considered, the uncoupled technique proposed is always about two or three times faster than the coupled approach.
\end{abstract}

Mathematics Subject Classification. 65N55, 65M55, 65F10, 92C30.

Received December 20, 2011. Revised May 13, 2012.

Published online 7 June 2013.

\section{INTRODUCTION}

The anisotropic Bidomain model describes the bioelectric activity of the cardiac tissue and consists of a system of a non-linear parabolic reaction-diffusion partial differential equation (PDE) and an elliptic linear PDE. This system models at macroscopic level the evolution of the transmembrane and extracellular electric potentials, $v$ and $u_{e}$ respectively, of the anisotropic cardiac tissue (macroscale). The evolution equation is coupled

Keywords and phrases. Operator splitting, multilevel preconditioners, parallel computing.

* The authors were partially supported by grants of MIUR (PRIN 2009Y4RC3B_003).

** The authors were partially supported by grants of MIUR (PRIN 2009Y4RC3B_002) and of INdAM (Istituto Nazionale di Alta Matematica, Roma, Italy).

1 Dipartimento di Matematica, Università di Pavia, Via Ferrata, 27100 Pavia, Italy. colli@imati.cnr.it

2 Dipartimento di Matematica, Università di Milano, Via Saldini 50, 20133 Milano, Italy. luca.pavarino@unimi.it; simone.scacchi@unimi.it 
through the non-linear reaction term with a stiff system of ordinary differential equations (ODEs), the so-called membrane model, describing the ionic currents through the cellular membrane (microscale).

The different space and time scales involved make the solution of the Bidomain system a very challenging scientific computing problem. In fact, the accurate approximation of the steep activation wavefront spreading through the heart tissue during the depolarization phase requires mesh sizes on the order of the tenth of millimeter and time step sizes on the order of the hundredth of millisecond, while the dimension of the heart muscle is on the order of centimeters and the duration of heart beat on the order of seconds. Thus, the discretization of the anisotropic Bidomain model in three-dimensional ventricular geometries of realistic size yields the solution of large scale (often exceeding $O\left(10^{7}\right)$ unknowns) and ill-conditioned linear systems at each time step.

Several approaches have been developed in order to reduce the high computational costs of the Bidomain model. Fully implicit methods in time, requiring the solution of non-linear systems at each time step, have been considered in the literature, see e.g. [24-26,41], but until now, due to high computational cost, they have not been used in large scale simulations. Alternatively, most previous works have considered IMEX time discretizations and/or operator splitting schemes, where the reaction and diffusion terms are treated separately, see e.g. $[4,6,8,14,20,22,28,35,38,45,47,53-55]$. The advantage of IMEX and operator splitting schemes is that they only require the solution of a linear system for the parabolic and elliptic PDEs at each time step. A further splitting approach consists in uncoupling the parabolic PDE from the elliptic one, see e.g. [1,53]. In [15], the authors have recently proved that Gauss-Seidel and Jacobi-like uncoupling approaches do not compromise the stability of the resulting time-stepping schemes.

Many different preconditioners have been proposed in order to devise efficient iterative solvers for the linear systems deriving from both the splitting techniques: Symmetric Successive Over Relaxation [32], block diagonal or triangular [6,17,33,34,52], optimized Schwarz [18], multigrid [1,33-35,46,54], multilevel Schwarz [29,30,40,42].

The aim of the present work is to introduce a novel uncoupling approach, based on solving twice the parabolic PDE and once the elliptic PDE at each time step. Three-dimensional numerical tests on both structured and unstructured meshes have been performed in order to show that the proposed uncoupled method has the same accuracy of the coupled strategy. Parallel numerical tests have also shown that the uncoupled technique is as scalable as the coupled one. Moreover, the conjugate gradient method preconditioned by Multilevel Hybrid Schwarz preconditioners converges faster for the linear systems deriving from the uncoupled method than from the coupled one. Finally, in all parallel numerical tests considered, the uncoupled technique proposed was always about 2.5-3 times faster than the coupled approach.

\section{The ANisotropic Bidomain MODEL}

The macroscopic Bidomain representation of the cardiac tissue volume $\Omega$ is obtained by considering the superposition of two anisotropic continuous media, the intra- (i) and extra- (e) cellular media, coexisting at every point of the tissue and separated by a distributed continuous cellular membrane; see e.g. [7,27,31] for a derivation of the Bidomain model from homogenization of cellular models. We recall that the cardiac tissue consists of an arrangement of fibers that rotate counterclockwise from epi- to endocardium, and that have a laminar organization modeled as a set of muscle sheets running radially from epi- to endocardium. The anisotropy of the intra- and extracellular media, related to the macroscopic arrangement of the cardiac myocytes in the fiber structure, is described by the anisotropic conductivity tensors $D_{i}(\mathbf{x})$ and $D_{e}(\mathbf{x})$, respectively, defined in (2) below.

\subsection{Continuous model}

We denote by $\Omega \subset \mathbb{R}^{3}$ the bounded physical region occupied by the cardiac tissue and introduce parabolicelliptic formulation of the Bidomain system. Given an applied extracellular current per unit volume $I_{\text {app }}^{\mathrm{e}}$ : $\Omega \times(0, T) \rightarrow \mathbb{R}$, and initial conditions $v_{0}: \Omega \rightarrow \mathbb{R}, w_{0}: \Omega \rightarrow \mathbb{R}^{N_{w}}$, find the the transmembrane potential $v: \Omega \times(0, T) \rightarrow \mathbb{R}$, extracellular potentials $u_{e}: \Omega \times(0, T) \rightarrow \mathbb{R}$, the gating variables $w: \Omega \times(0, T) \rightarrow \mathbb{R}^{N_{w}}$ and 
the ionic concentrations $c: \Omega \times(0, T) \rightarrow \mathbb{R}^{N_{c}}$ such that

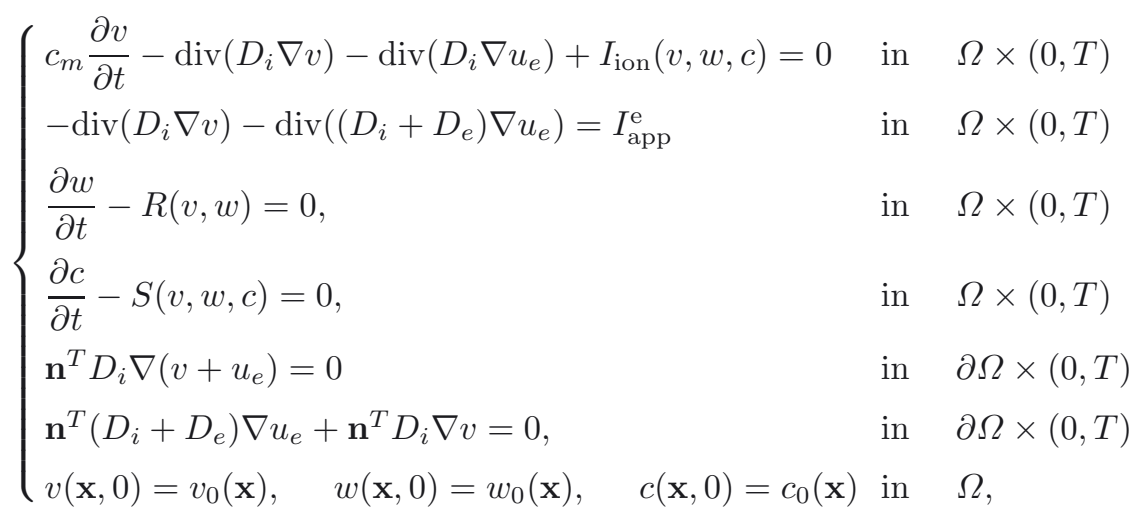

where $c_{m}$ is the membrane capacitance per unit volume. The applied extracellular current $I_{\text {app }}^{\mathrm{e}}$ must satisfy the compatibility condition $\int_{\Omega} I_{\mathrm{app}}^{\mathrm{e}} \mathrm{dx}=0$, and we impose the reference potential $\int_{\Omega} u_{e} \mathrm{dx}=0$. The non-linear reaction term $I_{\text {ion }}$ and the ODE system for the gating variables $w$ and the ionic concentrations $c$ are given by the ionic membrane model; see e.g. $[16,56]$ for recent reviews. Here we will consider the Luo-Rudy I (LR1) membrane model [21].

The conductivity tensors $D_{i}(\mathbf{x})$ and $D_{e}(\mathbf{x})$ at any point $\mathbf{x} \in \Omega$ are assumed orthotropic, thus defined as

$$
\begin{aligned}
D_{i, e}(\mathbf{x}) & =\sigma_{l}^{i, e} \mathbf{a}_{l}(\mathbf{x}) \mathbf{a}_{l}^{T}(\mathbf{x})+\sigma_{t}^{i, e} \mathbf{a}_{t}(\mathbf{x}) \mathbf{a}_{t}^{T}(\mathbf{x})+\sigma_{n}^{i, e} \mathbf{a}_{n}(\mathbf{x}) \mathbf{a}_{n}^{T}(\mathbf{x}) \\
& =\sigma_{l}^{i, e} I+\left(\sigma_{t}^{i, e}-\sigma_{l}^{i, e}\right) \mathbf{a}_{t}(\mathbf{x}) \mathbf{a}_{t}^{T}(\mathbf{x})+\left(\sigma_{n}^{i, e}-\sigma_{l}^{i, e}\right) \mathbf{a}_{n}(\mathbf{x}) \mathbf{a}_{n}^{T}(\mathbf{x}) .
\end{aligned}
$$

Here $\mathbf{a}_{l}(\mathbf{x}), \mathbf{a}_{t}(\mathbf{x}), \mathbf{a}_{n}(\mathbf{x})$, is a triplet of orthonormal principal axes with $\mathbf{a}_{l}(\mathbf{x})$ parallel to the local fiber direction, $\mathbf{a}_{t}(\mathbf{x})$ and $\mathbf{a}_{n}(\mathbf{x})$ tangent and orthogonal to the radial laminae, respectively, and both being transversal to the fiber axis (see e.g. LeGrice et al. [19]). Moreover, $\sigma_{l}^{i, e}, \sigma_{t}^{i, e}, \sigma_{n}^{i, e}$ are the conductivity coefficients in the intraand extracellular media measured along the corresponding directions $\mathbf{a}_{l}, \mathbf{a}_{t}, \mathbf{a}_{n}$.

\subsection{Variational formulation}

Let $V$ be the Sobolev space $H^{1}(\Omega)$, define the spaces

$$
\widetilde{V}=\left\{\psi \in V: \int_{\Omega} \psi=0\right\} \quad \text { and } \quad U=V \times \widetilde{V}=\{u=(\varphi, \psi): \varphi \in V, \psi \in \widetilde{V}\}
$$

define the usual $L^{2}$-inner product $(\varphi, \psi)=\int_{\Omega} \varphi \psi \mathrm{d} x \quad \forall \varphi, \psi \in L^{2}(\Omega)$, and the elliptic bilinear forms

$$
\begin{aligned}
a_{i, e}(\varphi, \psi) & =\int_{\Omega}(\nabla \varphi)^{T} D_{i, e}(x) \nabla \psi \mathrm{d} x, \\
a(\varphi, \psi) & =\int_{\Omega}(\nabla \varphi)^{T} D(x) \nabla \psi \mathrm{d} x \quad \forall \varphi, \psi \in H^{1}(\Omega),
\end{aligned}
$$

where $D=D_{i}+D_{e}$ is the bulk conductivity tensor.

The variational formulation of the Bidomain model reads as follows. Given $v_{0}, w_{0}, c_{0} \in L^{2}(\Omega), I_{\text {app }}^{\mathrm{e}} \in L^{2}(\Omega \times$ $(0, T))$, find $v \in L^{2}(0, T ; V), u_{e} \in L^{2}(0, T ; \widetilde{V}), w \in L^{2}\left(0, T ; L^{2}(\Omega)^{N_{w}}\right)$ and $c \in L^{2}\left(0, T ; L^{2}(\Omega)^{N_{c}}\right)$ such that 


$$
\begin{aligned}
\frac{\partial v}{\partial t} \in L^{2}(0, T ; V), \frac{\partial w}{\partial t} \in & L^{2}\left(0, T ; L^{2}(\Omega)^{N_{w}}\right), \frac{\partial c}{\partial t} \in L^{2}\left(0, T ; L^{2}(\Omega)^{N_{c}}\right) \text { and } \forall t \in(0, T) \\
& \left\{\begin{array}{l}
c_{m} \frac{\partial}{\partial t}(v, \hat{v})+a_{i}(v, \hat{v})+a_{i}\left(u_{e}, \hat{v}\right)+\left(I_{\text {ion }}(v, w, c), \hat{v}\right)=0 \quad \forall \hat{v} \in V \\
a_{i}\left(v, \hat{u}_{e}\right)+a\left(u_{e}, \hat{u}_{e}\right)=\left(I_{\text {app }}^{\mathrm{e}}, \hat{u}_{e}\right) \quad \forall \hat{u}_{e} \in \widetilde{V} \\
\frac{\partial}{\partial t}(w, \hat{w})-(R(v, w), \hat{w})=0, \quad \forall \hat{w} \in V, \\
\frac{\partial}{\partial t}(c, \hat{c})-(S(v, w, c), \hat{c})=0, \quad \forall \hat{c} \in V,
\end{array}\right.
\end{aligned}
$$

with the appropriate initial conditions in (1).

\section{DiscretizATION AND NUMERICAL METHODS}

\subsection{Space discretization}

System (3) is first discretized in space by the finite element method. Let $\mathcal{T}_{h}$ be a quasi-uniform triangulation of $\Omega$ having maximal diameter $h$ and $V_{h}$ be an associated conforming finite element space. In this work, we will consider isoparametric trilinear finite elements on exahedral meshes and linear finite elements on unstructured tetrahedral meshes. Once a finite element basis $\left\{\varphi_{l}\right\}_{l=1}^{N}$ of $V_{h}$ is chosen, we denote by $A_{i, e}=\left\{a_{l j}^{i, e}\right\}$ the symmetric intra- and extracellular stiffness matrices, and by $M=\left\{m_{l j}\right\}$ the mass matrix, with elements

$$
a_{l j}^{i, e}=\int_{\Omega} D_{i, e} \nabla \varphi_{j} \cdot \nabla \varphi_{l} \mathrm{dx}, \quad m_{l j}=\int_{\Omega} \varphi_{j} \varphi_{l} \mathrm{dx} .
$$

Applying a standard Galerkin procedure to (3) and using the finite element interpolants of $I_{\text {app }}^{\mathrm{e}}, I_{\mathrm{ion}}$ associated to the vectors of nodal values $\mathbf{I}_{\text {app }}^{\mathrm{e}}, \mathbf{I}_{\text {ion }}$, we obtain the following semi-discrete Bidomain problem, written in compact matrix form as

$$
\left\{\begin{array}{l}
c_{m} \mathbb{M} \frac{\mathrm{d}}{\mathrm{d} t}\left[\begin{array}{c}
\mathbf{v} \\
\mathbf{u}_{e}
\end{array}\right]+\mathbb{A}\left[\begin{array}{c}
\mathbf{v} \\
\mathbf{u}_{e}
\end{array}\right]+\left[\begin{array}{c}
M \mathbf{I}_{\mathrm{ion}}(\mathbf{v}, \mathbf{w}, \mathbf{c}) \\
\mathbf{0}
\end{array}\right]=\left[\begin{array}{c}
\mathbf{0} \\
M \mathbf{I}_{\mathrm{app}}^{\mathrm{e}}
\end{array}\right] \\
\frac{\mathrm{d} \mathbf{w}}{\mathrm{d} t}=\mathbf{R}(\mathbf{v}, \mathbf{w}) \\
\frac{\mathrm{d} \mathbf{c}}{\mathrm{d} t}=\mathbf{S}(\mathbf{v}, \mathbf{w}, \mathbf{c})
\end{array}\right.
$$

with block mass and stiffness matrices

$$
\mathbb{M}=\left[\begin{array}{ll}
M & 0 \\
0 & 0
\end{array}\right], \quad \mathbb{A}=\left[\begin{array}{cc}
A_{i} & A_{i} \\
A_{i} & A_{i}+A_{e}
\end{array}\right] .
$$

Here $\mathbf{v}, \mathbf{u}_{e}, \mathbf{w}=\left(\mathbf{w}_{1}, \ldots, \mathbf{w}_{N_{w}}\right)^{T}, \mathbf{c}=\left(\mathbf{c}_{1}, \ldots, \mathbf{c}_{N_{c}}\right)^{T}, \mathbf{R}(\mathbf{v}, \mathbf{w})=\left(R_{1}(\mathbf{v}, \mathbf{w}), \ldots, R_{N_{w}}(\mathbf{v}, \mathbf{w})\right)^{T}, \mathbf{S}(\mathbf{v}, \mathbf{w}, \mathbf{c})=$ $\left(S_{1}(\mathbf{v}, \mathbf{w}, \mathbf{c}), \ldots, S_{N_{c}}(\mathbf{v}, \mathbf{w}, \mathbf{c})\right)^{T}$, are the coefficient vectors of the finite element approximations of $u_{i}, u_{e}, v$, $w_{r}, c_{r}, R_{r}\left(v, w_{1}, \ldots, w_{N_{w}}\right), S_{r}\left(v, w_{1}, \ldots, w_{N_{w}}, c_{1}, \ldots, c_{N_{c}}\right)$, respectively. In our case of isoparametric trilinear or linear finite elements, these are the vectors of nodal values of these functions.

The mass matrix $M$ is diagonalized by the usual mass-lumping technique, where the diagonal elements are calculated as the row sums of the original mass matrix, that is equivalent to using a trapezoidal quadrature rule. This procedure preserves the optimal error estimates of the finite element method, see [49], Chapter 15, [37]. Moreover, since the Bidomain model exhibits a dominant reaction term, the mass-lumping technique increases the stability of the scheme avoiding spurious oscillations in the case of not too fine spatial meshes, see [39], Chapter 8. 


\subsection{Time discretization}

The time discretization of the Bidomain and Monodomain equations can be performed using either implicit, semi-implicit or explicit schemes, requiring accordingly vector updates or the solution of a non-linear or linear system. Fully implicit methods in time have been considered e.g. in [24-26,41]. The advantage of implicit methods is that they do not require stability constraints on the choice of the time step, but they are very expensive, because at each time step one has to solve a non-linear system. A good compromise between stability and efficiency is obtained using linear implicit methods, studied e. g. in $[9,10,24]$, which require at each time step the solution of 2-4 linear systems, or semi-implicit methods, studied e.g. in $[6,7,14,43,55]$. For a detailed comparative study on the stability and accuracy of several Bidomain time discretizations (implicit, semi-implicit, explicit), we refer to the recent work [14]. However, the most popular technique is based on operator splitting, i.e. on separating the diffusion operator, associated with conduction in the media, from the reaction operator, associated with the ionic current, gating and ionic concentrations dynamics. The advantage of splitting methods is to allow different numerical schemes for the diffusion and the reaction terms in order to maximize computational efficiency and eliminate complex dependency between variables. The disadvantage is a loss of accuracy, because the simultaneous dependency between variables is neglected. For the parabolic-elliptic formulation of the Bidomain model, a further splitting method consists in uncoupling the parabolic and elliptic equations, i.e. in solving sequentially the elliptic equation, followed by the parabolic one, or viceversa. Previous works have considered several variants of these splitting and/or uncoupling techniques. In particular, the uncoupled approach has been considered in $[1,22,35,36,45,47,51-54]$. The splitting of reaction and diffusion terms has been considered in $[35,53,54]$, while a three steps Strang splitting, with reaction - diffusion - reaction steps, has been considered in $[22,47,51]$. An additional approximation is made in $[35,54]$ by substituting the mass matrix with the identity. A recap of some splitting and uncoupling approaches can be found in the review papers $[5,20,28,53]$.

In this work, we compare two implicit-explicit (IMEX) strategies, both based on decoupling the ODEs from the PDEs and on treating the linear diffusion terms implicitly and the non-linear reaction terms explicitly. The implicit treatment of the diffusion term is needed in order to avoid a stability constraint on the time step $\Delta t$ induced by the fine mesh size $h$. Nevertheless, due to the explicit treatment of the reaction terms, stability could be preserved for a time step $\Delta t$ satisfying a condition of CFL type. To our knowledge, a theoretical and numerical investigation of stability properties of IMEX methods for the Bidomain model coupled to the LR1 membrane model is still lacking in literature. Some rigorous results on stability of IMEX methods for the Bidomain system coupled to the Fitzhugh-Nagumo membrane model are presented in [14].

- Coupled method. According to this strategy, the equations in (4) arising from the discretization of the PDEs are solved as a coupled system. Given $\mathbf{w}^{n}, \mathbf{c}^{n}, \mathbf{v}^{n}, \mathbf{u}_{\mathrm{e}}^{n}$ at the generic time step $n$ :

- we first solve the ODEs system by computing by Implicit Euler the new gating variables $\mathbf{w}^{n+1}$ and by Explicit Euler the new ionic concentrations $\mathbf{c}^{n+1}$,

- then we solve the PDEs system computing $\mathbf{v}^{n+1}$ and $\mathbf{u}_{\mathrm{e}}^{n+1}$.

Summarizing in formulae, given $\mathbf{w}^{n}, \mathbf{c}^{n}, \mathbf{v}^{n}, \mathbf{u}_{\mathrm{e}}^{n}$, the scheme is

$$
\begin{aligned}
\mathbf{w}^{n+1}-\Delta t \mathbf{R}\left(\mathbf{v}^{n}, \mathbf{w}^{n+1}\right) & =\mathbf{w}^{n} \\
& =\mathbf{c}^{n}+\Delta t \mathbf{S}\left(\mathbf{v}^{n}, \mathbf{w}^{n+1}, \mathbf{c}^{n}\right) \\
\mathbf{c}^{n+1} & =\frac{c_{m}}{\Delta t} \mathbb{M}\left[\begin{array}{c}
\mathbf{v}^{n} \\
\mathbf{u}_{\mathrm{e}}^{n}
\end{array}\right]+\left[\begin{array}{c}
-M \mathbf{I}_{\text {ion }}\left(\mathbf{v}^{n}, \mathbf{w}^{n+1}, \mathbf{c}^{n+1}\right) \\
M \mathbf{I}_{\text {app }}^{e, n+1}
\end{array}\right]
\end{aligned}
$$

As a consequence, at each time step, we solve one linear system with unknowns $\left(\mathbf{v}^{n+1}, \mathbf{u}_{\mathrm{e}}^{n+1}\right)$. Because the iteration matrix is symmetric positive semi-definite, the iterative method employed is the preconditioned conjugate gradient (PCG) method. Due to the ill-conditioning of the iteration matrix and the large number of unknowns required by realistic simulations of cardiac excitation in three-dimensional domains, a scalable and efficient preconditioner is required. In case of structured meshes we adopt the Multilevel Hybrid Schwarz 

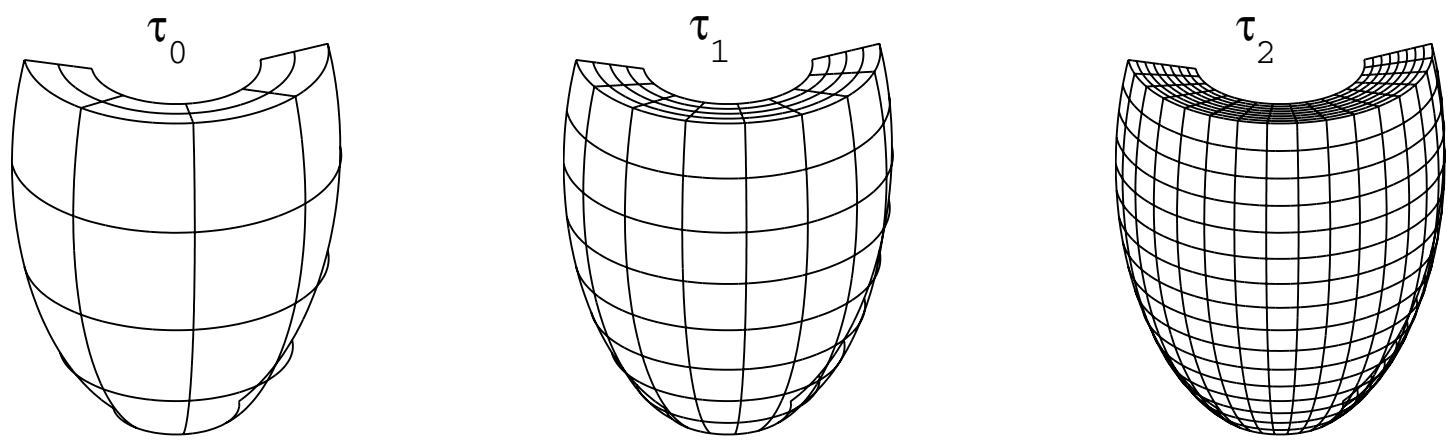

FiguRE 1. 3D example of nested meshes.

(MHS) preconditioner (see the next section), while in case of unstructured meshes we adopt the Block Jacobi (BJ) preconditioner.

- Uncoupled method. According to this second strategy, the two equations in (4) arising from the discretization of the PDEs are uncoupled by introducing the following predictor-corrector scheme. Given $\mathbf{w}^{n}$, $\mathbf{c}^{n}, \mathbf{v}^{n}, \mathbf{u}_{\mathrm{e}}^{n}$ at the generic time step $n$ :

- we first solve the ODEs system computing by Implicit Euler the new gating variables $\mathbf{w}^{n+1}$ and by Explicit Euler the new ionic concentrations $\mathbf{c}^{n+1}$,

- then we solve the parabolic equation finding an intermediate transmembrane potential $\widehat{\mathbf{v}}^{n+1}$,

- then we solve the elliptic equation computing $\mathbf{u}_{\mathrm{e}}^{n+1}$,

- and finally we update the transmembrane potential $\mathbf{v}^{n+1}$ by solving again the parabolic equation.

Summarizing in formulae, given $\mathbf{w}^{n}, \mathbf{c}^{n}, \mathbf{v}^{n}, \mathbf{u}_{\mathrm{e}}^{n}$, the scheme is

$$
\begin{aligned}
\mathbf{w}^{n+1}-\Delta t \mathbf{R}\left(\mathbf{v}^{n}, \mathbf{w}^{n+1}\right) & =\mathbf{w}^{n} \\
\mathbf{c}^{n+1} & =\mathbf{c}^{n}+\Delta t \mathbf{S}\left(\mathbf{v}^{n}, \mathbf{w}^{n+1}, \mathbf{c}^{n}\right) \\
\left(\frac{c_{m}}{\Delta t} M+A_{i}\right) \widehat{\mathbf{v}}^{n+1} & =\frac{c_{m}}{\Delta t} M \mathbf{v}^{n}-A_{i} \mathbf{u}_{\mathrm{e}}^{n}-M \mathbf{I}_{\mathrm{ion}}\left(\mathbf{v}^{n}, \mathbf{w}^{n+1}, \mathbf{c}^{n+1}\right) \\
\left(A_{i}+A_{e}\right) \mathbf{u}_{\mathrm{e}}^{n+1} & =-A_{i} \widehat{\mathbf{v}}^{n+1}+M \mathbf{I}_{\mathrm{app}}^{e, n+1} \\
\left(\frac{c_{m}}{\Delta t} M+A_{i}\right) \mathbf{v}^{n+1} & =\frac{c_{m}}{\Delta t} M \mathbf{v}^{n}-A_{i} \mathbf{u}_{\mathrm{e}}^{n+1}-M \mathbf{I}_{\mathrm{ion}}\left(\mathbf{v}^{n}, \mathbf{w}^{n+1}, \mathbf{c}^{n+1}\right) .
\end{aligned}
$$

As a consequence, at each time step we solve twice the linear system with matrix $\frac{c_{m}}{\Delta t} M+A_{i}$ arising from the parabolic equation, and once the linear system with matrix $A_{i}+A_{e}$ arising from the elliptic equation. Both linear systems are solved by the PCG method, since the matrices are symmetric positive definite in the parabolic case and semi-definite in the elliptic case. The preconditioner used for the parabolic system is the BJ, because the related matrix is well-conditioned, while that used for the elliptic system is the MHS in case of structured meshes and the BJ in case of unstructured meshes.

This uncoupled method adds a further correction step to the standard uncoupled method by solving again the parabolic equation in $\mathbf{v}^{n}$ by a semi-implicit method. In the standard uncoupled method, the first parabolic correction can be solved explicitly as e.g. in $[36,45]$ or by a semi-implicit method as e.g. in $[1,45]$.

\subsection{The Multilevel Hybrid Schwarz preconditioner}

In this section we introduce the Multilevel Hybrid Schwarz preconditioner used in the numerical tests and proposed in [40] (see also [11,12,44,50,57] for further details on this method) to overcome the computational limits arising from the solution of the Bidomain model.

Let $\mathcal{T}_{i}, i=0, \ldots, \ell-1$ be a family of $\ell$ nested triangulations of $\Omega$, coarsening from $\ell-1$ to 0 , hence $\mathcal{T}_{0}$ represents the coarsest level of discretization and $\mathcal{T}_{\ell-1}$ the finest one (see Fig. 1 for 3D examples of nested 
triangulations). Let us define the matrix $\mathrm{A}=\frac{c_{m}}{\Delta t} \mathbb{M}+\mathbb{A}$ in the coupled method case and $\mathrm{A}=A_{i}+A_{e}$ in the uncoupled method case, and the restriction operators $\mathrm{R}^{(i)}$ from level $\mathcal{T}_{i+1}$ to level $\mathcal{T}_{i}$. With these definitions, set the matrices

$$
\mathrm{A}^{(i)}:=\mathrm{R}^{(i)} \mathrm{AR}^{(i)^{T}}
$$

thus $\mathrm{A}^{(\ell-1)}=\mathrm{A}$. We then decompose $\Omega$ into $N$ overlapping subdomains, hence each grid $\mathcal{T}_{i}$ is decomposed into $N$ overlapping subgrids $\Omega_{k}^{(i)}$ for $k=1, \ldots, N$, such that the overlap $\delta^{(i)}$ at level $i=1, \ldots, \ell-1$ is equal to the mesh size $h^{(i)}$ of the grid $\mathcal{T}_{i}$. Let $R_{k}^{(i)}$, for $k=1, \ldots, N$, be the restriction operators from $\mathcal{T}_{i}$ to $\Omega_{k}^{(i)}$, that is $R_{k}^{(i)}$ returns the vector of all the coefficients associated with domain $\Omega_{k}^{(i)}$. Define the matrix

$$
\mathrm{A}_{k}^{(i)}:=\mathrm{R}_{k}^{(i)} \mathrm{A}^{(i)} \mathrm{R}_{k}^{(i)}
$$

i.e. the subblock matrix of $\mathrm{A}^{(i)}$ associated with domain $\Omega_{k}^{(i)}$. The action of the $\ell$-level hybrid Schwarz preconditioner $(\operatorname{MHS}(\ell))$ on a given fine level residual $\mathbf{r}^{(\ell-1)}$ of the PCG iteration is computed as:

$$
\begin{aligned}
& \mathbf{u}^{(\ell-1)} \leftarrow \sum_{k=1}^{N} \mathrm{R}_{k}^{(\ell-1)^{T}} \mathrm{~A}_{k}^{(\ell-1)^{-1}} \mathrm{R}_{k}^{(\ell-1)} \mathbf{r}^{(\ell-1)} \\
& \mathbf{r}^{(\ell-2)} \leftarrow \mathrm{R}^{(\ell-2)}\left(\mathbf{r}-\mathrm{A}^{(\ell-1)} \mathbf{u}^{(\ell-1)}\right) \\
& \mathbf{u}^{(\ell-2)} \leftarrow \sum_{k=1}^{N} \mathrm{R}_{k}^{(\ell-2)^{T}} \mathrm{~A}_{k}^{(\ell-2)^{-1}} \mathrm{R}_{k}^{(\ell-2)} \mathbf{r}^{(\ell-2)} \\
& \mathbf{r}^{(\ell-3)} \leftarrow \mathrm{R}^{(\ell-3)}\left(\mathbf{r}^{(\ell-2)}-\mathrm{A}^{(\ell-2)} \mathbf{u}^{(\ell-2)}\right) \\
& \cdots \\
& \mathbf{u}^{(0)} \leftarrow \mathrm{A}^{(0)^{-1}} \mathbf{r}^{(0)} \\
& \mathbf{u}^{(1)} \leftarrow \mathbf{u}^{(1)}+\mathrm{R}^{(0)^{T}} \mathbf{u}^{(0)} \\
& \mathbf{u}^{(1)} \leftarrow \mathbf{u}^{(1)}+\sum_{k=1}^{N} \mathrm{R}_{k}^{(1)^{T}} \mathrm{~A}_{k}^{(1)^{-1}} \mathrm{R}_{k}^{(1)}\left(\mathbf{r}^{(1)}-\mathrm{A}^{(1)} \mathbf{u}^{(1)}\right) \\
& \cdots \\
& \mathbf{u}^{(\ell-1)} \leftarrow \mathbf{u}^{(\ell-1)}+\mathrm{R}^{(\ell-2)^{T}} \mathbf{u}^{(\ell-2)} \\
& \mathbf{u}^{(\ell-1)} \leftarrow \mathbf{u}^{(\ell-1)}+\sum_{k=1}^{N} \mathrm{R}_{k}^{(\ell-1)^{T}} \mathrm{~A}_{k}^{(\ell-1)^{-1}} \mathrm{R}_{k}^{(\ell-1)}\left(\mathbf{r}^{(\ell-1)}-\mathrm{A}^{(\ell-1)} \mathbf{u}^{(\ell-1)}\right) \\
& \mathbf{u} \leftarrow \mathbf{u}^{(\ell-1)}
\end{aligned}
$$

We remark that $\operatorname{MHS}(\ell)$ is additive on each level among the subdomains and multiplicative among the levels.

\section{Numerical Results}

In this section, we present the results of parallel numerical experiments performed on the Linux Cluster IBM SP6 of the Cineca Consortium (www.cineca.it). Our FORTRAN code is based on the parallel library PETSc [2,3], from the Argonne National Laboratory.

The Bidomain system coupled to the LR1 model is integrated by the coupled and uncoupled Implicit-Explicit methods described in the previous sections. The values of the coefficients and parameters in the LR1 model are given in the original paper [21]. The linear systems at each time step are solved by the preconditioned conjugate gradient (PCG) method, using as stopping criterion a $10^{-6}$ reduction of the relative residual $l^{2}$-norm. In case of structured meshes, for the coupled method the linear system is preconditioned by the MHS(4) preconditioner, while for the uncoupled method the elliptic linear system is preconditioned by the MHS(4) preconditioner and 


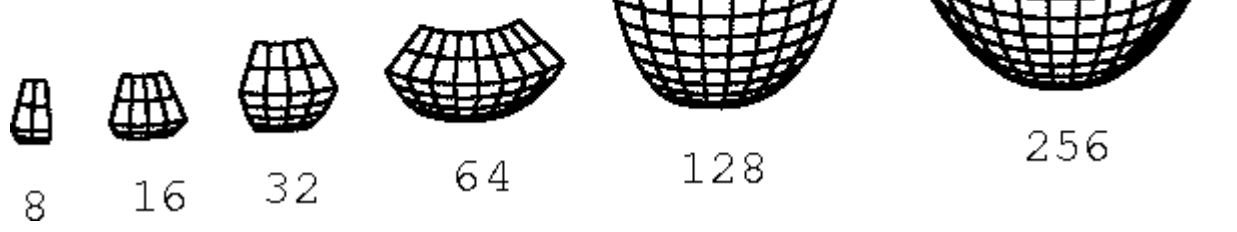

Figure 2. Portions of ellipsoidal domain decomposed in 8, 16, 32, 64, 128, 256 subdomains for scaled speedup test.

the parabolic linear systems are preconditioned by the BJ preconditioner. In case of unstructured meshes, all the linear systems are preconditioned by the BJ preconditioner, because up to now we have not implemented a multilevel method for unstructured tetrahedral meshes. In all cases, ILU(0) local solvers are used for the local problems on the subdomains.

Domain geometry and fiber structure. In the structured mesh case, the domain $\Omega$ is either a cartesian slab or the image of a cartesian slab using ellipsoidal coordinates, yielding a portion of truncated ellipsoid (see Fig. 2). The family of truncated ellipsoids is described by the parametric equations

$$
\begin{cases}x=a(r) \cos \theta \cos \phi & \phi_{\min } \leq \phi \leq \phi_{\max } \\ y=b(r) \cos \theta \sin \phi & \theta_{\min } \leq \theta \leq \theta_{\max } \\ z=c(r) \sin \theta & 0 \leq r \leq 1\end{cases}
$$

where $a(r)=a_{1}+r\left(a_{2}-a_{1}\right), b(r)=b_{1}+r\left(b_{2}-b_{1}\right), c(r)=c_{1}+r\left(c_{2}-c_{1}\right)$, and $a_{1}=1.5, a_{2}=2.7, b_{1}=$ $1.5, b_{2}=2.7, c_{1}=4.4, c_{2}=5$ are given coefficients (all in $\mathrm{cm}$ ) determining the main axes of the ellipsoid. In the unstructured mesh case, the domain $\Omega$ is a cartesian slab of dimension $2 \times 2 \times 0.5 \mathrm{~cm}^{3}$ discretized by a tetrahedral grid generated using TETGEN [48] and partitioned into subdomains using METIS [23]. The fibers rotate intramurally linearly with the depth for a total amount of $120^{\circ}$ proceeding counterclockwise from epicardium to endocardium. More precisely, in a local ellipsoidal reference system $\left(\mathbf{e}_{\phi}, \mathbf{e}_{\theta}, \mathbf{e}_{r}\right)$, the fiber direction $\mathbf{a}_{l}(\mathbf{x})$ at a point $\mathbf{x}$ is given by

$$
\mathbf{a}_{l}(\mathbf{x})=\mathbf{e}_{\phi} \cos \alpha(r)+\mathbf{e}_{\theta} \sin \alpha(r), \quad \text { with } \quad \alpha(r)=\frac{2}{3} \pi(1-r)-\frac{\pi}{4}, \quad 0 \leq r \leq 1 .
$$

Conductivity coefficients. The values of the conductivity coefficients in (2) used in all the numerical tests are the following:

$$
\begin{array}{ll}
\sigma_{l}^{i}=3 \times 10^{-3} \Omega^{-1} \mathrm{~cm}^{-1} & \sigma_{l}^{\mathrm{e}}=2 \times 10^{-3} \Omega^{-1} \mathrm{~cm}^{-1} \\
\sigma_{t}^{i}=3.1525 \times 10^{-4} \Omega^{-1} \mathrm{~cm}^{-1} & \sigma_{t}^{\mathrm{e}}=1.3514 \times 10^{-3} \Omega^{-1} \mathrm{~cm}^{-1} \\
\sigma_{n}^{i}=3.1525 \times 10^{-5} \Omega^{-1} \mathrm{~cm}^{-1} & \sigma_{n}^{\mathrm{e}}=6.757 \times 10^{-4} \Omega^{-1} \mathrm{~cm}^{-1}
\end{array}
$$

Mesh hierarchy. We denote the cartesian mesh used to discretize our domains by $\mathcal{T}=\mathcal{T}_{i} \cdot \mathcal{T}_{j} \cdot \mathcal{T}_{k}$, indicating the number of elements in each coordinate direction. This notation applies to both fine and coarse meshes. In the case of the MHS preconditioner, the coarse meshes are constructed by progressively halving the number of elements in each coordinate direction.

Stimulation site, initial and boundary conditions. The depolarization process is started by applying a stimulus of $I_{\text {app }}=-200 \mathrm{~mA} / \mathrm{cm}^{3}$ lasting $1 \mathrm{~ms}$ on the face of the domain modeling the endocardial surface. The initial conditions are at resting values for all the potentials and LR1 gating variables, while the boundary conditions are for insulated tissue. In all simulations, the fine mesh size is $h=0.01 \mathrm{~cm}$. The time step size is $\Delta t=0.05 \mathrm{~ms}$. 
TABle 1. Test 1. Accuracy in time of coupled, uncoupled and standard uncoupled methods. Relative errors $\operatorname{err}_{v}=\frac{\left\|v(\cdot, 10)-v_{\text {ref }}(\cdot, 10)\right\|_{L^{2}(\Omega)}}{\left\|v_{\text {ref }}(\cdot, 10)\right\|_{L^{2}(\Omega)}}, \operatorname{err}_{\text {ue }}=\frac{\left\|u_{e}(\cdot, 10)-u_{e, \text { ref }}(\cdot, 10)\right\|_{L^{2}(\Omega)}}{\left\|u_{\mathrm{e}, \text { ref }}(\cdot, 10)\right\|_{L^{2}(\Omega)}}$ and $\operatorname{err}_{\mathrm{at}}=$ $\frac{\left\|a t(\cdot)-a t_{\mathrm{ref}}(\cdot)\right\|_{L} \infty(\Omega)}{\left\|a t_{\mathrm{ref}}(\cdot)\right\|_{L^{\infty}(\Omega)}}$.

\begin{tabular}{|l|ccc|ccc|ccc|}
\hline$\Delta t$ & \multicolumn{3}{|c|}{ coupled } & \multicolumn{3}{c|}{ uncoupled } & \multicolumn{3}{c|}{ standard uncoupled } \\
& $e r r_{v}$ & $e r r_{\text {ue }}$ & $e r r_{\text {at }}$ & $e r r_{v}$ & $e r r_{\text {ue }}$ & $e r r_{\text {at }}$ & $e r r_{v}$ & $e r r_{\text {ue }}$ & $e r r_{\text {at }}$ \\
\hline 0.1 & 0.1053 & 0.1559 & 0.0320 & 0.1227 & 0.1745 & 0.0368 & 0.1560 & 0.2216 & 0.0496 \\
0.05 & 0.0678 & 0.1013 & 0.0198 & 0.0797 & 0.1164 & 0.0222 & 0.0905 & 0.1314 & 0.0283 \\
0.025 & 0.0344 & 0.0523 & 0.0097 & 0.0396 & 0.0592 & 0.0108 & 0.0420 & 0.0626 & 0.0135 \\
0.0125 & 0.0127 & 0.0194 & 0.0035 & 0.0145 & 0.0219 & 0.0039 & 0.0156 & 0.0239 & 0.0052 \\
\hline
\end{tabular}

Remark 4.1. In Tables $2,3,4$ and 5, the condition number $\left(\kappa_{2}\right)$, the maximum and minimum eigenvalues $\left(\lambda_{M}\right.$ and $\lambda_{m}$ ), the PCG iteration count (it) and CPU time (time) of the coupled method column refer to the unique linear system to be solved at each time step, while $\kappa_{2}, \lambda_{M}, \lambda_{m}$ and $i t$ of the uncoupled method column refer to the linear system deriving from the elliptic equation and time is the sum of CPU times needed for solving the elliptic and the two parabolic linear systems.

\subsection{Test 1: accuracy in time}

In this first test, the coupled and uncoupled methods are compared in terms of accuracy with respect to the time step size. The domain considered is a three-dimensional slab of dimension $0.64 \times 0.64 \times 0.48 \mathrm{~cm}^{3}$, discretized by a structured grid of $64 \times 64 \times 48 Q_{1}$ finite elements, distributed among 8 processors. With this choice of parameters, at each time step the coupled method yields the solution of one linear system with 414050 degrees of freedom $(d o f)$, while the uncoupled method yields the solution of three linear systems with 207025 dof, two related to the parabolic equation and one to the elliptic equation. We also consider the standard uncoupled method without the third parabolic correction, recalled in Section 3.2, that yields the solution of two linear systems with 207025 dof, one for the parabolic equation and one for the elliptic equation. The simulation is run until the tissue is completely depolarized. Different time step sizes are considered. Because an exact solution is not available, as a reference solution we take the one computed by the coupled method with $\Delta t=0.00625 \mathrm{~ms}$. Three errors are computed, one for the spatial distribution of the transmembrane potential at $t=10 \mathrm{~ms}$, i.e.

$$
\operatorname{err}_{v}=\frac{\left\|v(\cdot, 10)-v_{\mathrm{ref}}(\cdot, 10)\right\|_{L^{2}(\Omega)}}{\left\|v_{\mathrm{ref}}(\cdot, 10)\right\|_{L^{2}(\Omega)}},
$$

one for the spatial distribution of the extracellular potential at $t=10 \mathrm{~ms}$, i.e.

$$
\operatorname{err}_{\mathrm{ue}}=\frac{\left\|u_{e}(\cdot, 10)-u_{\mathrm{e}, \mathrm{ref}}(\cdot, 10)\right\|_{L^{2}(\Omega)}}{\left\|u_{\mathrm{e}, \mathrm{ref}}(\cdot, 10)\right\|_{L^{2}(\Omega)}},
$$

and one for the spatial distribution of the activation time, an important physiological quantity defined as the unique instant at $(x)$ for which $v(x, a t(x))=-50 \mathrm{mV}$ during the upstroke phase of the action potential, i.e.

$$
e r r_{\mathrm{at}}=\frac{\left\|a t(\cdot)-a t_{\mathrm{ref}}(\cdot)\right\|_{L^{\infty}(\Omega)}}{\left\|a t_{\mathrm{ref}}(\cdot)\right\|_{L^{\infty}(\Omega)}} .
$$

All the previous integrals are computed expressing the functions in terms of the finite element basis.

We remark that both the coupled and uncoupled methods are first order accurate in time. The results reported in Table 1 document that, although in terms of relative errors the uncoupled method is slightly less accurate than the coupled one, from a practical viewpoint the difference is not significant. Figure 3 displays the endocardial 

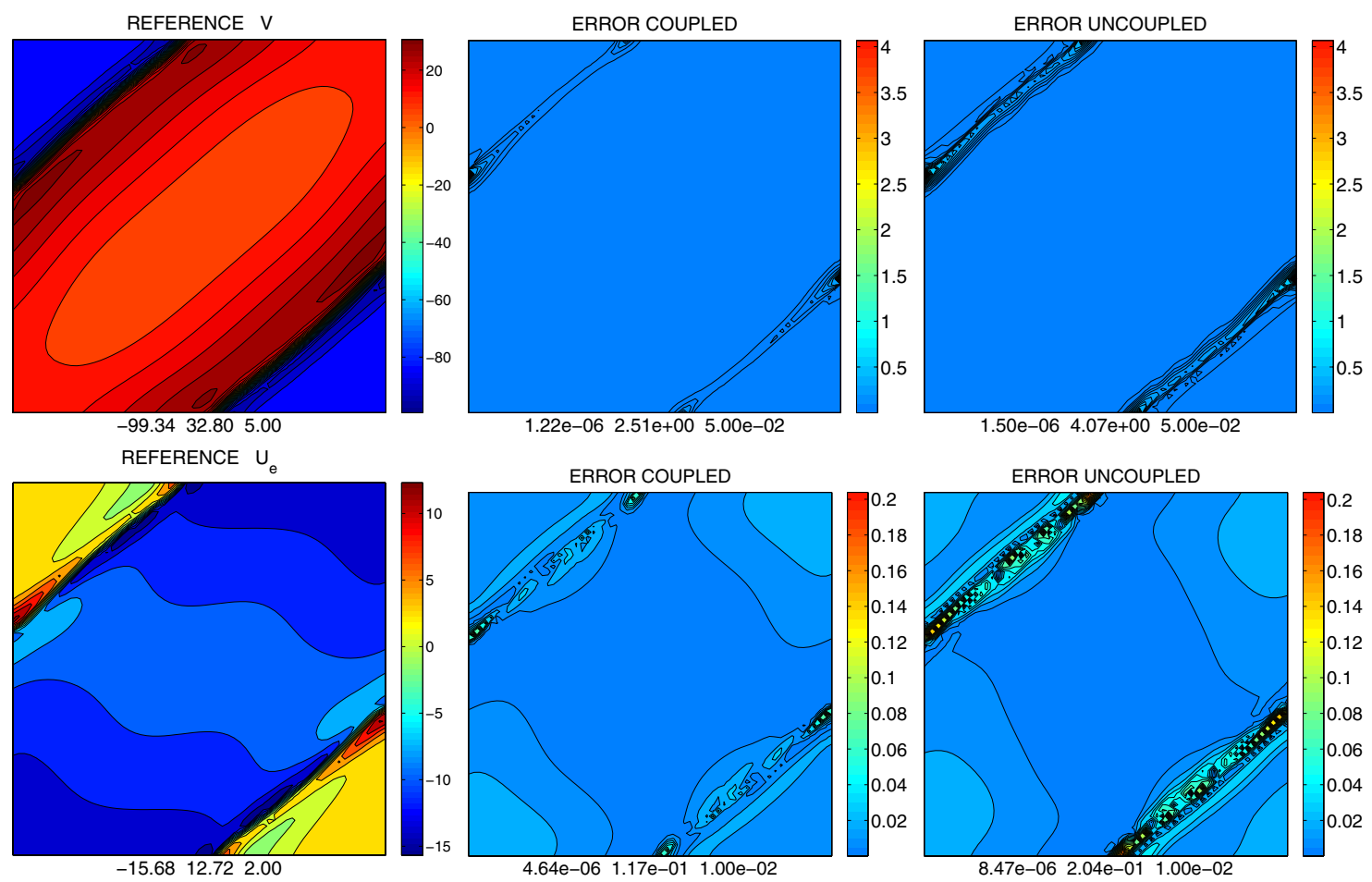

Figure 3. Test 1. Accuracy in time of coupled and uncoupled methods. First row. Endocardial transmembrane potential distribution $10 \mathrm{~ms}$ after the beginning of stimulation for the reference solution (left), error $\left|v_{\text {coup }}(\cdot, 10)-v_{\text {ref }}(\cdot, 10)\right| /\left|v_{\text {ref }}(\cdot, 10)\right|$ between the coupled method solution with $\Delta t=0.05 \mathrm{~ms}$ and the reference solution (middle), error $\mid v_{\text {uncoup }}(\cdot, 10)-$ $v_{\text {ref }}(\cdot, 10)|/| v_{\text {ref }}(\cdot, 10) \mid$ between the uncoupled method solution with $\Delta t=0.05 \mathrm{~ms}$ and the reference solution (right). Second row. Endocardial extracellular potential distribution $10 \mathrm{~ms}$ after the beginning of stimulation for the reference solution (left), error $\mid u_{\mathrm{e}, \mathrm{coup}}(\cdot, 10)-$ $u_{\mathrm{e}, \mathrm{ref}}(\cdot, 10)|/| u_{\mathrm{e}, \mathrm{ref}}(\cdot, 10) \mid$ between the coupled method solution with $\Delta t=0.05 \mathrm{~ms}$ and the reference solution (middle), error $\left|u_{\mathrm{e}, \text { uncoup }}(\cdot, 10)-u_{\mathrm{e}, \text { ref }}(\cdot, 10)\right| /\left|u_{\mathrm{e}, \text { ref }}(\cdot, 10)\right|$ between the uncoupled method solution with $\Delta t=0.05 \mathrm{~ms}$ and the reference solution (right). Below each contour plot are reported the minimum, maximum and step in $m V$ of the displayed map.

(bottom section of the slab) transmembrane potential distribution at $t=10 \mathrm{~ms}$ of the reference solution (left), of the solution computed by the coupled method with $\Delta t=0.05 \mathrm{~ms}$ (middle) and of the solution computed by the uncoupled method with $\Delta t=0.05 \mathrm{~ms}$ (right). Figure 4 reports the isochrones of activation time on the endocardial, midmyocardial and epicardial sections of the slab computed from the reference solution, the coupled method solution and the uncoupled method solution with $\Delta t=0.05 \mathrm{~ms}$. From a visual comparison, even with a quite large time step size $(0.05 \mathrm{~ms})$, the coupled and uncoupled solutions are almost indistinguishable between them, and from the reference solution.

We have also considered a standard uncoupled method recalled in Section 3.2. The last three columns of Table 1 show that as expected the standard uncoupled method is slightly less accurate than our uncoupled method, particularly for larger time step sizes.

\subsection{Test 2: dependence of the linear solvers on the time step size}

We compare here the linear solvers performance of the coupled and uncoupled methods with varying time step size $\Delta t$. The three-dimensional domain is the portion of a truncated ellipsoid discretized by a grid of 

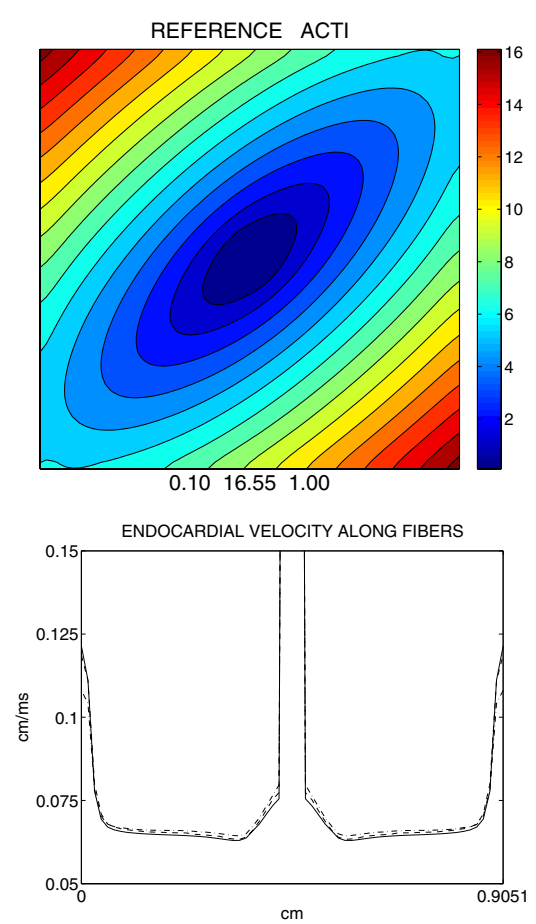

ERROR COUPLED

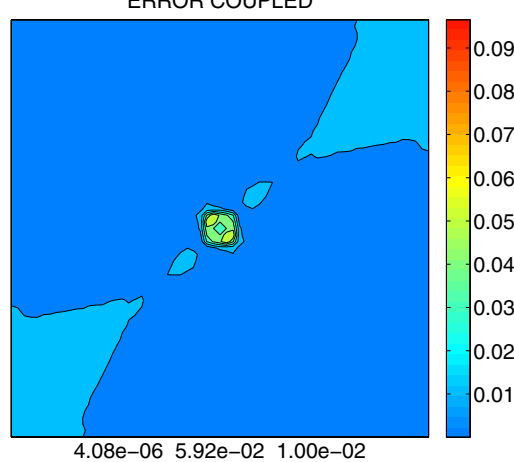

ENDOCARDIAL VELOCITY ACROSS FIBERS

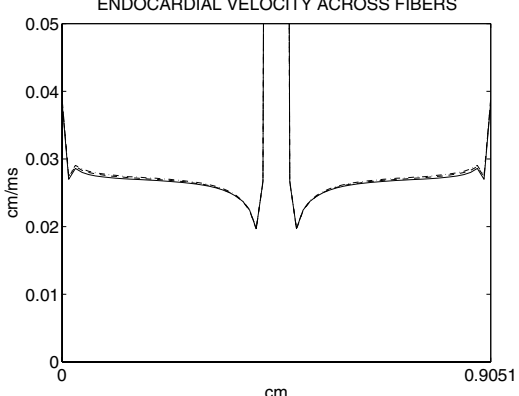

ERROR UNCOUPLED

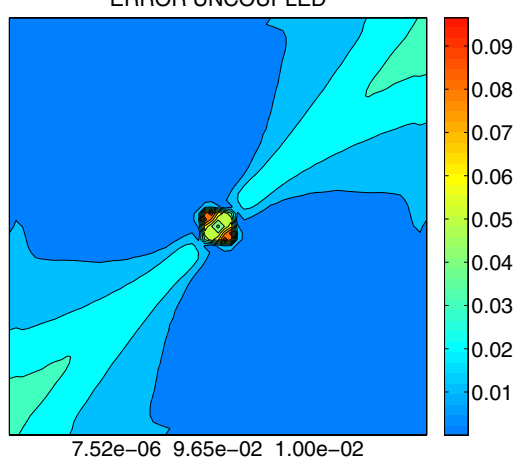

TRANSMURAL VELOCITY

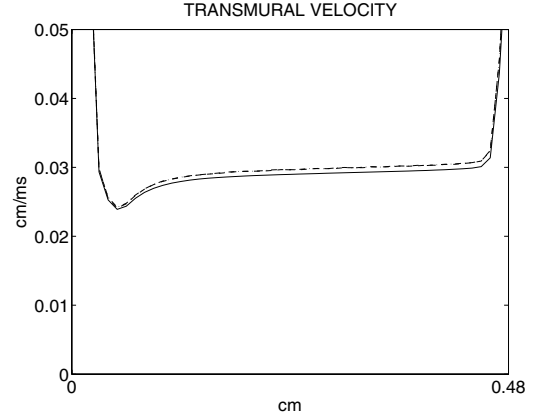

Figure 4. Test 1. Accuracy in time of coupled and uncoupled methods. First row. Endocardial activation time isochrones computed from the reference solution (left), error $\mid a t_{\text {coup }}(\cdot)-$ $a t_{\text {ref }}(\cdot)|/| a t_{\text {ref }}(\cdot) \mid$ between the endocardial activation time isochrones computed from the coupled method solution with $\Delta t=0.05 \mathrm{~ms}$ and from the reference solution (middle), error $\left|a t_{\text {uncoup }}(\cdot)-a t_{\text {ref }}(\cdot)\right| /\left|a t_{\text {ref }}(\cdot)\right|$ between the endocardial activation time isochrones computed from the uncoupled method with $\Delta t=0.05 \mathrm{~ms}$ and from the reference solution (right). Below each contour plot are reported the minimum, maximum and step in $m s$ of the displayed map. Second row. Endocardial activation wavefront velocity along fibers (left), across fibers (middle) and transmural activation wavefront velocity (right) of the reference solution (continuous line), coupled method solution (dashed line) and uncoupled method solution (dashed-dotted line).

$384 \times 384 \times 96 Q_{1}$ finite elements, distributed among 128 processors, see Figure 2 . With this choice of parameters, at each time step the coupled method yields the solution of one linear system with $28.7 \mathrm{M}$ unknowns, while the uncoupled method yields the solution of three linear systems with $14.4 \mathrm{M}$ unknowns. The simulation is run for $2 \mathrm{~ms}$ during the excitation phase of the heart beat. Different time step sizes are considered. In both coupled and uncoupled cases, Ttime reported in Table 2 represents the total simulation time.

The results displayed in Table 2 show that, in terms of mathematical quantities, i.e. $\kappa_{2}, \lambda_{M}, \lambda_{m}$, it, the performances of coupled and uncoupled linear solvers are comparable and do not depend on the time step size. In terms of CPU time instead, the uncoupled method is about three times faster than the coupled method, irrespectively of the time step size.

We have also computed the average timings per time step of the standard uncoupled method, obtaining respectively 2.6, 1.7, 1.6, $1.6 \mathrm{msec}$ for the same time step sizes $\Delta t=0.1,0.05,0.025,0.0125$ of Table 2. Except the first, these timings are only marginally better than the average timings of our uncoupled method. In particular, for decreasing time step sizes the differences between the average timings of the two uncoupled methods decreases toward zero, i.e. the cost of the additional parabolic correction of our uncoupled method 
TABle 2. Test 2. Dependence of linear system MHS(4) solvers on the time step size for coupled and uncoupled methods. Number of processors $=128$, dof $=28.7$ M. Average condition number $\left(\kappa_{2}\right)$, extreme eigenvalues $\left(\lambda_{M}, \lambda_{m}\right)$, PCG iteration count $(i t)$ and CPU time in seconds (time) per time step. Ttime is the total CPU simulation time in seconds. See Remark 4.1.

\begin{tabular}{|l|ccc|ccc|}
\hline$\Delta t$ & \multicolumn{3}{|c|}{ coupled } & \multicolumn{3}{c|}{ uncoupled } \\
& $\kappa_{2}=\lambda_{M} / \lambda_{m}$ & it & time (Ttime) & $\kappa_{2}=\lambda_{M} / \lambda_{m}$ & it & time $($ Ttime $)$ \\
\hline 0.1 & $1.21=1.02 / 0.84$ & 5 & $8.2(164.9)$ & $1.23=1.03 / 0.84$ & 5 & $3.1(62.7)$ \\
0.05 & $1.21=1.01 / 0.84$ & 5 & $6.0(238.7)$ & $1.22=1.03 / 0.84$ & 5 & $1.9(77.8)$ \\
0.025 & $1.21=1.01 / 0.84$ & 5 & $5.9(476.9)$ & $1.22=1.02 / 0.84$ & 5 & $1.8(147.3)$ \\
0.0125 & $1.20=1.01 / 0.84$ & 5 & $5.7(921.2)$ & $1.21=1.02 / 0.84$ & 5 & $1.7(281.6)$ \\
\hline
\end{tabular}

TABle 3. Test 3. Weak scaling of MHS(4) solvers on ellipsoidal structured meshes. Average condition number $\left(\kappa_{2}\right)$, extreme eigenvalues $\left(\lambda_{M}, \lambda_{m}\right)$, PCG iteration count $(i t)$ and CPU time in seconds (time) per time step. The scaled speedup is reported in brackets. See Remark 4.1.

\begin{tabular}{|l|c|ccc|ccccc|}
\hline procs & dof & \multicolumn{4}{|c|}{ coupled } & \multicolumn{4}{c|}{ uncoupled } \\
& & $\kappa_{2}=\lambda_{M} / \lambda_{m}$ & it & time & $\kappa_{2}=\lambda_{M} / \lambda_{m}$ & it & time \\
\hline 8 & $1.8 \mathrm{M}$ & $1.64=1.05 / 0.64$ & 6 & 5.9 & $(1.0)$ & $1.36=1.09 / 0.80$ & 5 & 1.9 & $(1.0)$ \\
16 & $3.6 \mathrm{M}$ & $1.64=1.05 / 0.64$ & 6 & 6.5 & $(1.8)$ & $1.36=1.09 / 0.80$ & 5 & 2.1 & $(1.8)$ \\
32 & $7.2 \mathrm{M}$ & $1.65=1.07 / 0.65$ & 6 & 6.5 & $(3.6)$ & $1.33=1.07 / 0.81$ & 5 & 2.1 & $(3.6)$ \\
64 & $14.4 \mathrm{M}$ & $1.65=1.07 / 0.65$ & 6 & 6.7 & $(7.0)$ & $1.33=1.08 / 0.81$ & 5 & $2.2(6.9)$ \\
128 & $28.7 \mathrm{M}$ & $1.60=1.06 / 0.66$ & 6 & $6.9(13.7)$ & $1.29=1.07 / 0.82$ & 5 & $2.3(13.2)$ \\
256 & $57.4 \mathrm{M}$ & $1.60=1.06 / 0.66$ & 6 & $7.0(27.0)$ & $1.29=1.07 / 0.82$ & 5 & $2.6(23.3)$ \\
512 & $114.7 \mathrm{M}$ & $1.55=1.05 / 0.68$ & 6 & $7.4(51.0)$ & $1.27=1.06 / 0.84$ & 5 & $2.9(41.9)$ \\
\hline
\end{tabular}

becomes negligible. For both this reason and the lower accuracy shown in Table 1, in the rest of the numerical tests we will not consider the standard uncoupled method.

\subsection{Test 3: weak scaling on ellipsoidal domains, structured mesh}

The coupled and uncoupled linear solvers are compared here in a scaled speedup test on ellipsoidal deformed domains, discretized by structured meshes. The number of subdomains (and processors) is increased from 8 to 512, forming increasing ellipsoidal domains $\Omega$ as shown in Figure 2. The fine mesh is chosen so as to keep the local mesh size on each subdomain fixed at $48 \cdot 48 \cdot 48$. With these choices, the global size of the discrete Bidomain system increases from about 1.8 million dof for the smallest domain with 8 subdomains to 114.7 million dof for the largest domain with 512 subdomains. The physical dimensions of the increasing cartesian slabs are chosen so that the fine mesh size $h$ is kept fixed to the value $h=0.01 \mathrm{~cm}$. The simulation is run for 10 time steps of $0.05 \mathrm{~ms}$ during the depolarization phase, which is the most intense computationally.

The results reported in Table 3 clearly show that, since the MHS(4) preconditioner is employed, both the coupled and uncoupled methods are scalable. In fact, all mathematical quantities (condition number, extreme eigenvalues, PCG iteration count) seem to approach constant values when increasing the number of subdomains. Also the CPU times scale quite well, because they only increase of about a factor $1.2-1.5$ from 8 to 512 processors, while the global problem increases of a factor 64 . For better clarity, the condition number and CPU times are also plotted in Figure 5. The scaled speedup, defined as

$$
\text { speedup }(\text { procs })=\frac{\text { time }(8)}{\text { time }(\text { procs })} \frac{\text { procs }}{8},
$$

is reported in brackets in Table 3 and plotted in Figure 6. 

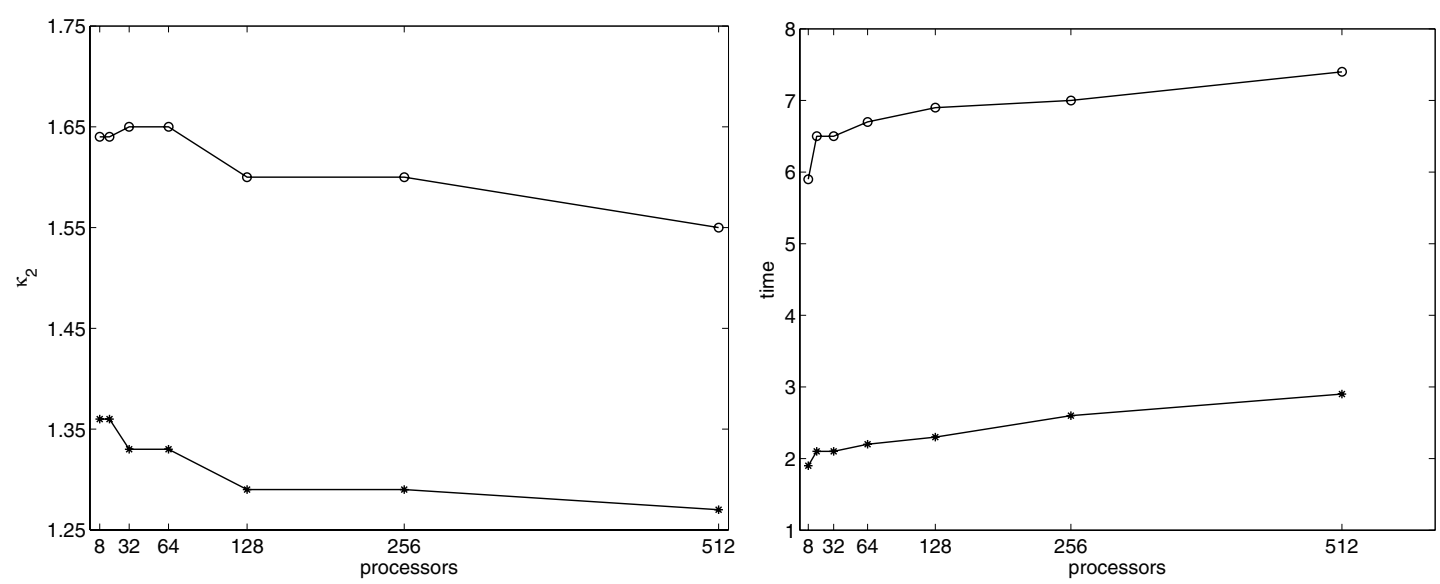

FiguRE 5. Test 3. Weak scaling of MHS(4) solvers on ellipsoidal structured meshes. Condition numbers (left) and CPU times (right) with respect to the number of processors for coupled (o) and uncoupled $(*)$ methods.

\begin{tabular}{|r|r|ccc|ccc|}
\hline procs & local dof & \multicolumn{3}{|c|}{ coupled } & \multicolumn{2}{c|}{ uncoupled } \\
& & $\kappa_{2}=\lambda_{M} / \lambda_{m}$ & it & time & $\kappa_{2}=\lambda_{M} / \lambda_{m}$ & it & time \\
\hline 8 & 304336 & $1.5 \mathrm{e}+4=2.37 / 1.6 \mathrm{e}-4$ & 350 & $23.8(1.0)$ & $1.4 \mathrm{e}+4=2.35 / 1.7 \mathrm{e}-4$ & 346 & $9.6(1.0)$ \\
16 & 152168 & $1.6 \mathrm{e}+4=2.47 / 1.6 \mathrm{e}-4$ & 356 & $15.1(1.6)$ & $1.5 \mathrm{e}+4=2.44 / 1.6 \mathrm{e}-4$ & 351 & $6.5(1.5)$ \\
32 & 76084 & $1.6 \mathrm{e}+4=2.47 / 1.6 \mathrm{e}-4$ & 358 & $9.7(2.4)$ & $1.5 \mathrm{e}+4=2.44 / 1.6 \mathrm{e}-4$ & 352 & $3.9(2.5)$ \\
64 & 38042 & $1.6 \mathrm{e}+4=2.47 / 1.5 \mathrm{e}-4$ & 358 & $5.8(4.1)$ & $1.5 \mathrm{e}+4=2.40 / 1.6 \mathrm{e}-4$ & 352 & $2.3(4.2)$ \\
128 & 19021 & $1.6 \mathrm{e}+4=2.47 / 1.5 \mathrm{e}-4$ & 359 & $3.7(6.4)$ & $1.5 \mathrm{e}+4=2.40 / 1.6 \mathrm{e}-4$ & 353 & $1.7(5.6)$ \\
\hline
\end{tabular}

TABLE 4. Test 4. Strong scaling of BJ solvers on a slab unstructured tetrahedral mesh of 7.5M elements with 1.2 M dof. Average condition number $\left(\kappa_{2}\right)$, extreme eigenvalues $\left(\lambda_{M}, \lambda_{m}\right)$, PCG iteration count (it) and CPU time in seconds (time) per time step. The standard speedup is reported in brackets. See Remark 4.1.

\subsection{Test 4: strong scaling on a slab domain, unstructured mesh}

In this standard speedup test, we compare the performance of coupled and uncoupled methods on an unstructured grid. The cardiac domain $\Omega$ is a three-dimensional slab discretized by a $P_{1}$ unstructured finite elements mesh of 7549221 tetrahedra and 1217351 nodes. With this choice of parameters, at each time step the coupled method yields the solution of one linear system with 2434702 unknowns, while the uncoupled method yields the solution of three linear systems with 1217351 unknowns. Since this is a strong scaling test, the mesh is fixed, while the number of subdomains (= number of processors) increases from 8 to 128. In this way, the number of dof per subdomain is reduced when the number of subdomains is increased. The simulation is run for $0.5 \mathrm{~ms}$ during the excitation phase, thus the total amount of time steps is 10 .

Table 4 reports the average condition number, extreme eigenvalues, PCG iterations count and CPU time per time step. The speedup, defined with respect to the 8 processors run as

$$
\text { speedup }(\text { procs }):=\frac{\text { Ttime }(8)}{\text { Ttime }(\text { procs })}
$$

is reported in brackets and plotted in Figure 6. Since the not scalable Block Jacobi preconditioner is employed for solving each linear system, the results reported in Table 4 show that the linear solvers of both the coupled and 

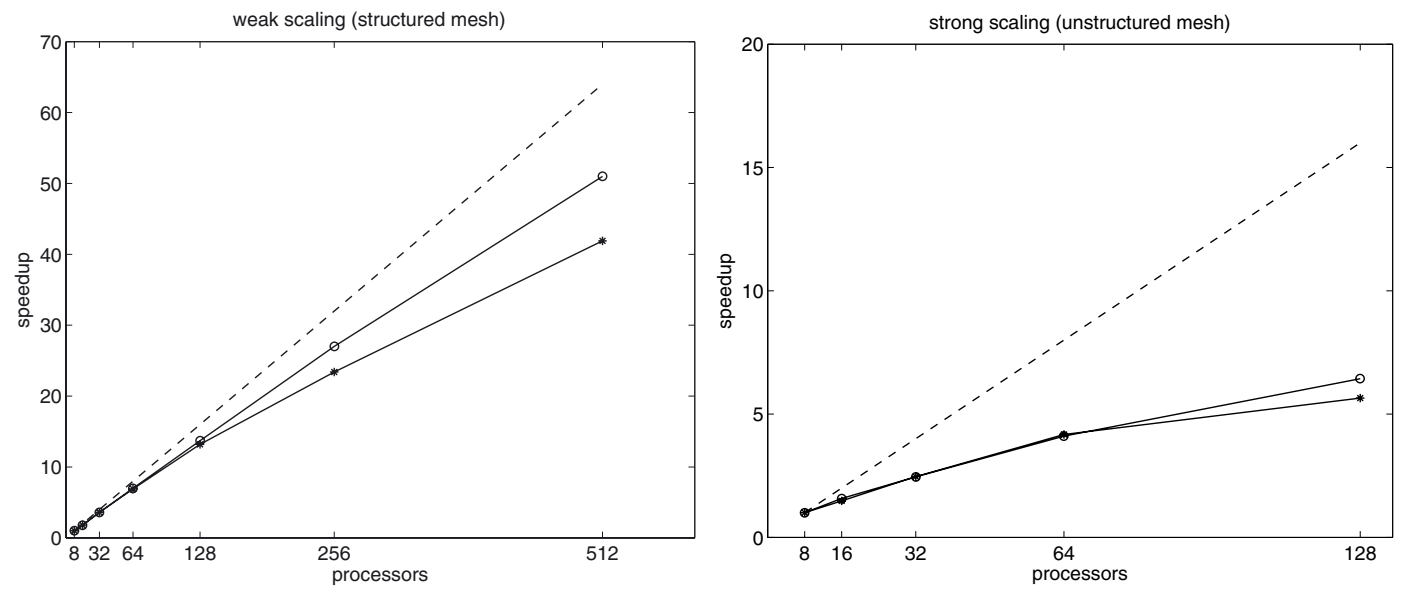

Figure 6. Scaled speedup (left) relative to Test 3 (structured mesh, MHS(4) solver) and standard speedup (right) relative to Test 4 (unstructured mesh, BJ solver). Comparison between coupled (o) and uncoupled $(*)$ methods. The ideal speedup is plotted in dashed line.

TABLE 5. Test 5. Comparison of coupled and uncoupled methods for MHS(4) and BJ solvers on a structured mesh and BJ solvers on an unstructured mesh during a whole heartbeat simulation. Average PCG iteration count (it) and CPU time (time) per time step, total PCG iteration count (Tit) and CPU time (Ttime). See Remark 4.1.

\begin{tabular}{|c|c|c|rrrr|rrrr|}
\hline mesh & prec. & dof & \multicolumn{4}{|c|}{ coupled } & \multicolumn{4}{c|}{ uncoupled } \\
& & & it & Tit & time & Ttime & it & Tit & time & Ttime \\
\hline structured & MHS(4) & $7.2 \mathrm{M}$ & 11 & 16976 & 38.9 & 58590.5 & 6 & 9453 & 16.0 & 24118.3 \\
structured & BJ & $7.2 \mathrm{M}$ & 355 & 534415 & 202.3 & 304622.0 & 249 & 375014 & 50.6 & 76154.8 \\
\hline unstructured & BJ & $2.4 \mathrm{M}$ & 453 & 827750 & 13.2 & 24076.9 & 454 & 828674 & 5.7 & 10347.8 \\
\hline
\end{tabular}

uncoupled methods do not scale as well as in the previous test, as expected. Nevertheless, for what concerns the efficiency comparison between the coupled and uncoupled methods, the present standard speedup test confirms that the uncoupled method is faster (about 2.4 times) than the coupled method also on unstructured meshes.

\subsection{Test 5: comparison between coupled and uncoupled methods on a complete cardiac cycle simulation}

In this last test, we first compare the coupled vs. the uncoupled solver on a complete heartbeat (400 ms) in a portion of ellipsoid, modeling a wedge of ventricular tissue, discretized by a $Q_{1}$ structured finite element grid of $193 \times 193 \times 97$ elements $(7226306$ dof $)$. Then we extend the comparison of the coupled vs. the uncoupled solver on a complete heartbeat (400 ms) in a slab domain, discretized by a $P_{1}$ unstructured finite element grid of 7549221 tetrahedra and 1217351 nodes (2 434702 dof). Because the mesh generator used (TETGEN [48]) is serial, we were not able to generate an unstructured mesh as large as the structured one, hence the two tests are not directly comparable. In the case of the structured mesh, we consider both the MHS(4) and the BJ preconditioners, while in the case of the unstructured mesh only the BJ preconditioner. The simulations are run on 32 processors. The time step size is changed according to the adaptive strategy described in [6].

The results reported in Table 5 confirm that the uncoupled method is faster than the coupled one, more precisely 2.4-4 times faster in case of the structured mesh and 2.3 faster in case of the unstructured mesh. 

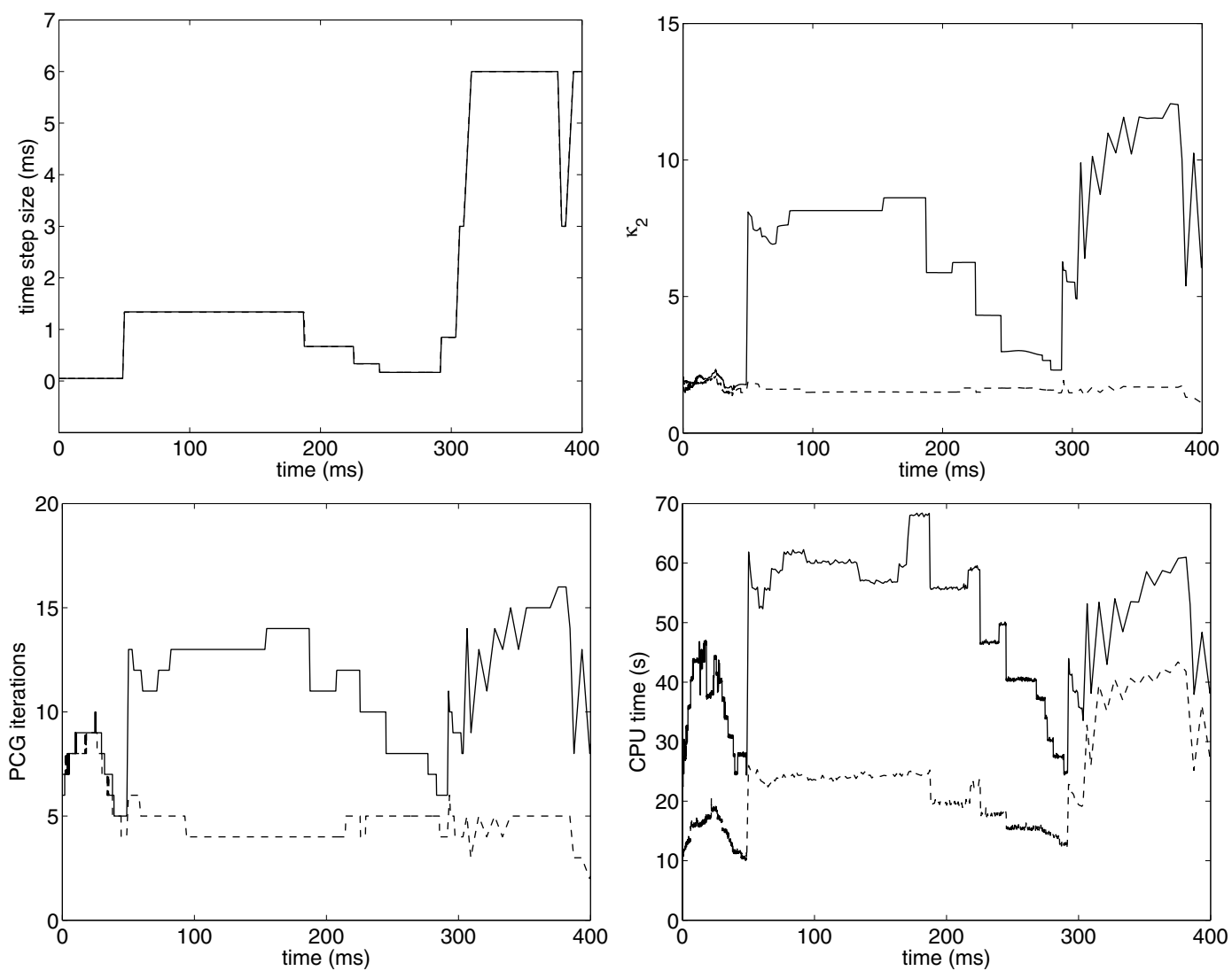

FiguRe 7. Test 5: comparison between coupled (continuous line) and uncoupled (dashed line) methods with MHS(4) preconditioner on a complete cardiac cycle simulation on a structured mesh. Time step size in ms (first row, left), condition number (first row, right), PCG iterations (second row, left) and CPU times (second row, right) with respect to time evolution.

The detailed time evolution of time step size, condition number, PCG iterations and CPU time per time step is reported in Figures 7, 8 and 9, where it is clear that in case of structured meshes, the reduction of CPU time for the uncoupled method is due to the reduction of PCG iterations particularly during the plateau and repolarization phases, from 50 to $350 \mathrm{~ms}$. In case of unstructured mesh, the PCG iterations of coupled and uncoupled methods are almost the same, but the uncoupled method is faster because the linear systems have half the dof of those of the coupled method.

\section{Conclusion}

We have proposed an uncoupled solver for the parabolic-elliptic Bidomain system, based on solving twice the parabolic PDE and once the elliptic PDE at each time step, and we have compared it with a standard coupled solver. Firstly, we have performed three-dimensional numerical tests on both structured and unstructured meshes and we have shown that the proposed uncoupled method has the same accuracy of the coupled strategy. In addition, parallel numerical tests have shown that the uncoupled technique is as scalable as the coupled one. In case of structured meshes, the conjugate gradient method preconditioned by Multilevel Hybrid Schwarz preconditioners exhibits a faster convergence in solving the linear systems associated to the uncoupled method 

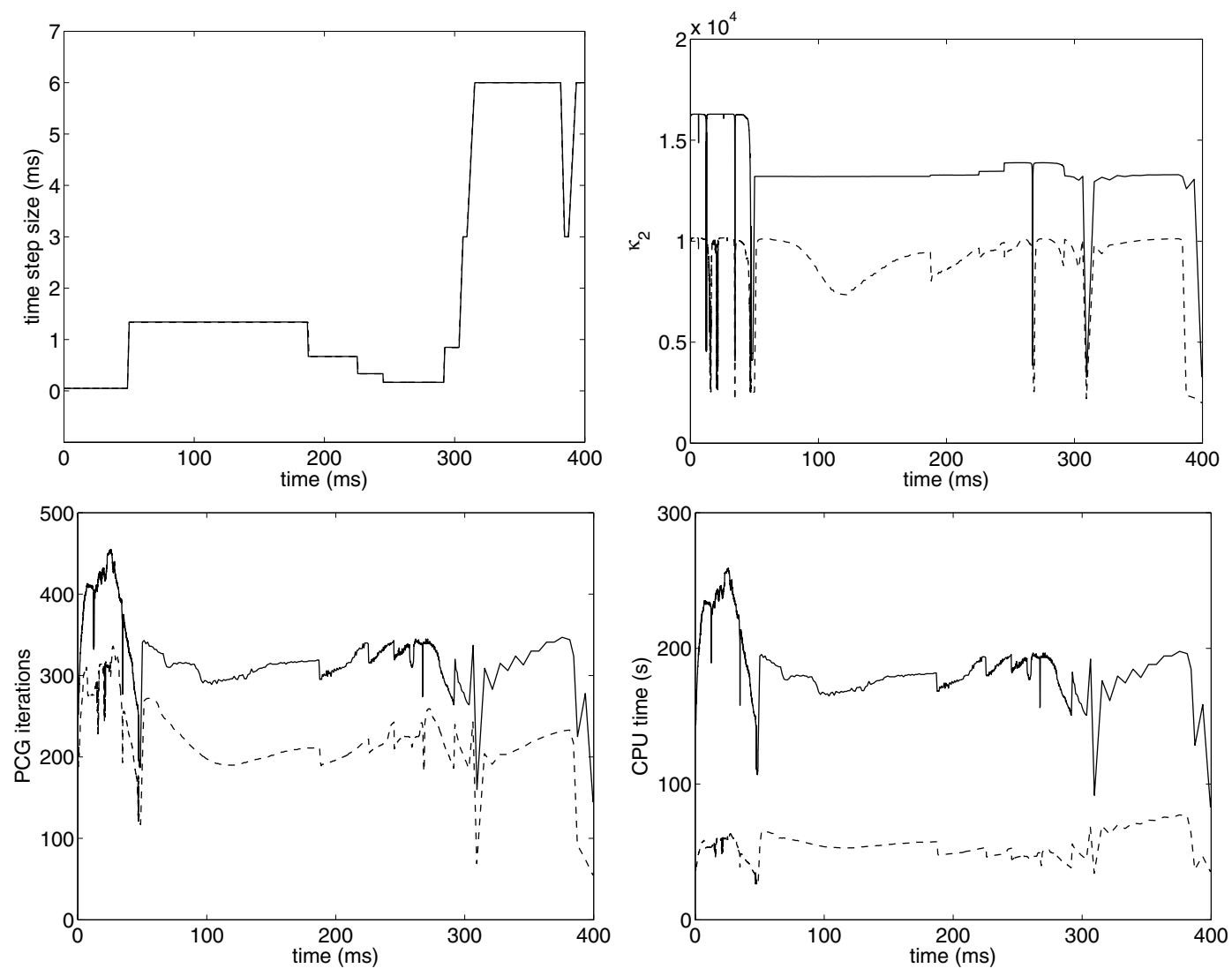

Figure 8. Test 5: comparison between coupled (continuous line) and uncoupled (dashed line) methods with BJ preconditioner on a complete cardiac cycle simulation on a structured mesh. Time step size in ms (first row, left), condition number (first row, right), PCG iterations (second row, left) and CPU times (second row, right) with respect to time evolution.

than those associated to the coupled one. More precisely, the uncoupled solver is more insensitive than the coupled one on the choice of large time step size during the plateau and repolarization phases, because the matrix associated to the elliptic equation does not involve the time step size parameter. For what concerns efficiency, all the parallel numerical tests considered have shown that the uncoupled technique is about 2.5-3 times faster than the coupled approach, employing the same PCG relative tolerances for both the coupled and uncoupled systems. A recent study [45] has shown that solving the coupled system can be more efficient than the uncoupled one when considering different uncoupled methods without the last parabolic correction step, that requires different PCG relative tolerances in order to achieve the same level of accuracy for the coupled and uncoupled approaches. We have also performed some tests with a standard uncoupled method without the last parabolic correction step and we have found that also this variant is $2-3$ times faster than the coupled approach, although less accurate than our uncoupled method. A direct comparison between the two uncoupled approaches is made difficult also by additional differences such as the use of Algebraic Multigrid preconditioners, unstructured meshes for realistic cardiac geometries, presence of an extracardiac bath where a grounded electrode Dirichlet boundary condition is imposed. The study of how these differences affect the solver efficiency will require future investigation. 

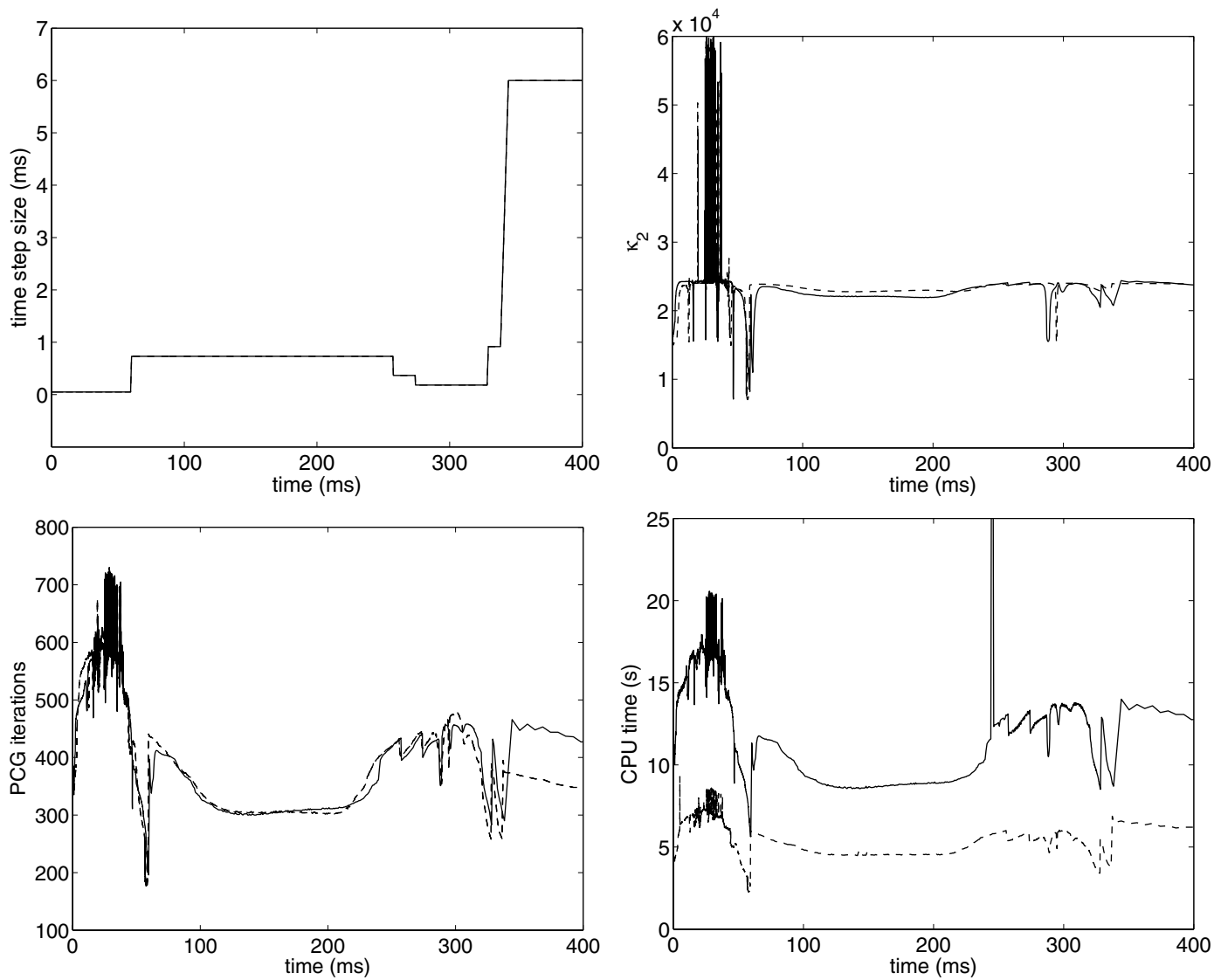

Figure 9. Test 5: comparison between coupled (continuous line) and uncoupled (dashed line) methods with BJ preconditioner on a complete cardiac cycle simulation on an unstructured mesh. Time step size in ms (first row, left), condition number (first row, right), PCG iterations (second row, left) and CPU times (second row, right) with respect to time evolution.

\section{REFERENCES}

[1] T.M. Austin, M.L. Trew and A.J. Pullan, Solving the cardiac Bidomain equations for discontinuous conductivities. IEEE Trans. Biomed. Eng. 53 (2006) 1265-1272.

[2] S. Balay, K. Buschelman, W.D. Gropp, D. Kaushik, M. Knepley, L. Curfman McInnes, B.F. Smith and H. Zhang, PETSc Users Manual.Tech. Rep. ANL-95/11 - Revision 2.1.5, Argonne National Laboratory (2002).

[3] S. Balay, K. Buschelman, W.D. Gropp, D. Kaushik, M. Knepley, L. Curfman McInnes, B.F. Smith and H. Zhang, PETSc home page. http://www.mcs.anl.gov/petsc (2001).

[4] M. Boulakia, S. Cazeau, M.A. Fernandez, J.-F. Gerbeau and N. Zemzemi, Mathematical modeling of electrocardiograms: a numerical study. Ann. Biomed. Eng. 38 (2010) 1071-1097.

[5] R.H. Clayton, O. Bernus, E.M. Cherry, H. Dierckx, F.H. Fenton, L. Mirabella, A.V. Panfilov, F.B. Sachse, G. Seemann and H. Zhang, Models of cardiac tissue electrophysiology: Progress, challenges and open questions. Progr. Biophys. Molec. Biol. 104 (2011) 22-48.

[6] P. Colli Franzone and L.F. Pavarino, A parallel solver for reaction-diffusion systems in computational electrocardiology. Math. Mod. Meth. Appl. Sci. 14 (2004) 883-911.

[7] P. Colli Franzone, L.F. Pavarino and S. Scacchi, Mathematical and numerical methods for reaction-diffusion models in electrocardiology, in Modeling of Physiological flows, edited by D. Ambrosi, A. Quarteroni and G. Rozza. Springer (2011) $107-142$.

[8] P. Colli Franzone, L.F. Pavarino and B. Taccardi, Simulating patterns of excitation, repolarization and action potential duration with cardiac bidomain and monodomain models. Math. Biosci. 197 (2005) 33-66.

[9] P. Colli Franzone, P. Deuflhard, B. Erdmann, J. Lang and L.F. Pavarino, Adaptivity in space and time for reaction-diffusion systems in Electrocardiology. SIAM J. Sci. Comput. 28 (2006) 942-962. 
[10] P. Deuflhard, B. Erdmann, R. Roitzsch and G.T. Lines, Adaptive finite element simulation of ventricular fibrillation dynamics. Comput. Visual. Sci. 12 (2009) 201-205.

[11] M. Dryja, M.V. Sarkis and O.B. Widlund, Multilevel Schwarz methods for elliptic problems with discontinuous coefficients in three dimensions. Numer. Math. 72 (1996) 313-348.

[12] M. Dryja and O.B. Widlund, Multilevel additive methods for elliptic finite element problems. Parallel algorithms for partial differential equations (Kiel 1990) Notes Numer. Fluid Mech. 31 (1991) 58-69.

[13] M. Dryja and O.B. Widlund, Domain decomposition algorithms with small overlap. SIAM J. Sci. Comput. 15 (1994) 604-620.

[14] M. Ethier and Y. Bourgault, Semi-implicit time-discretization schemes for the Bidomain model. SIAM J. Numer. Anal. 46 (2008) 2443-2468.

[15] M.A. Fernandez and N. Zemzemi, Decoupled time-marching schemes in computational cardiac electrophysiology and ECG numerical simulation. Math. Biosci. 226 (2010) 58-75.

[16] M. Fink, S.A. Niederer, E.M. Cherry, F.H. Fenton, J.T. Koivumaki, G. Seemann, T. Rudiger, H. Zhang, F.B. Sachse, D. Beard, E.J. Crampin and N.P. Smith, Cardiac cell modelling: observations from the heart of the cardiac physiome project. Prog. Biophys. Mol. Biol. 104 (2011) 2-21.

[17] L.G. Giorda, L. Mirabella, F. Nobile, M. Perego and A. Veneziani, A model-based block-triangular preconditioner for the Bidomain system in electrocardiology. J. Comput. Phys. 228 (2009) 3625-3639.

[18] L. Gerardo Giorda, M. Perego and A. Veneziani, Optimized Schwarz coupling of Bidomain and Monodomain models in electrocardiology. Math. Model. Numer. Anal. 45 (2011) 309-334.

[19] I.J. LeGrice, B.H. Smaill, L.Z. Chai, S.G. Edgar, J.B. Gavin and P.J. Hunter, Laminar structure of the heart: ventricular myocyte arrangement and connective tissue architecture in the dog. Amer. J. Physiol. Heart Circ. Physiol. 269 (1995) H571H582.

[20] S. Linge, J. Sundnes, M. Hanslien, G.T. Lines and A. Tveito, Numerical solution of the bidomain equations. Philos. Trans. R. Soc. A 367 (2009) 1931-1950.

[21] C. Luo and Y. Rudy, A model of the ventricular cardiac action potential: depolarization, repolarization, and their interaction. Circ. Res. 68 (1991) 1501-1526.

[22] K.-A. Mardal, B.F. Nielsen, X. Cai and A. Tveito, An order optimal solver for the discretized bidomain equations. Numer. Linear Algebra Appl. 14 (2007) 83-98.

[23] G. Karypis and V. Kumar, MeTis: Unstructured Graph Partitioning and Sparse Matrix Ordering System, Version 4.0. http://www.cs.umn.edu/ metis/. University of Minnesota, Minneapolis, MN (2009).

[24] M. Munteanu and L.F. Pavarino, Decoupled Schwarz algorithms for implicit discretization of nonlinear Monodomain and Bidomain systems. Math. Mod. Meth. Appl. Sci. 19 (2009) 1065-1097.

[25] M. Munteanu, L.F. Pavarino and S. Scacchi. A scalable Newton-Krylov-Schwarz method for the Bidomain reaction-diffusion system. SIAM J. Sci. Comput. 31 (2009) 3861-3883.

[26] M. Murillo and X.-C. Cai, A fully implicit parallel algorithm for simulating the non-linear electrical activity of the heart. Numer. Linear Algebra Appl. 11 (2004) 261-277.

[27] J.S. Neu and W. Krassowska, Homogenization of syncytial tissues. Crit. Rev. Biomed. Eng. 21 (1993) $137-199$.

[28] P. Pathmanathan, M.O. Bernabeu, R. Bordas, J. Cooper, A. Garny, J.M. Pitt-Francis, J.P. Whiteley and D.J. Gavaghan, A numerical guide to the solution of the bidomain equations of cardiac electrophysiology. Progr. Biophys. Molec. Biol. 102 (2010) 136-155.

[29] L.F. Pavarino and S. Scacchi, Multilevel additive Schwarz preconditioners for the Bidomain reaction-diffusion system. SIAM J. Sci. Comput. 31 (2008) 420-443.

[30] L.F. Pavarino and S. Scacchi, Parallel Multilevel Schwarz and Block Preconditioners for the Bidomain Parabolic-Parabolic and Parabolic-Elliptic Formulations. SIAM J. Sci. Comput. 33 (2011) 1897-1919.

[31] M. Pennacchio, G. Savaré and P.C. Franzone. Multiscale modeling for the bioelectric activity of the heart. SIAM J. Math. Anal. 37 (2006) 1333-1370.

[32] M. Pennacchio and V. Simoncini, Efficient algebraic solution of reaction-diffusion systems for the cardiac excitation process. J. Comput. Appl. Math. 145 (2002) 49-70.

[33] M. Pennacchio and V. Simoncini, Algebraic multigrid preconditioners for the bidomain reaction-diffusion system. Appl. Numer. Math. 59 (2009) 3033-3050.

[34] M. Pennacchio and V. Simoncini, Fast structured AMG preconditioning for the bidomain model in electrocardiology. SIAM J. Sci. Comput. 33 (2011) 721-745.

[35] G. Plank, M. Liebmann, R. Weber dos Santos, E.J. Vigmond and G. Haase, Algebraic Multigrid Preconditioner for the Cardiac Bidomain Model. IEEE Trans. Biomed. Eng. 54 (2007) 585-596.

[36] M. Potse, B. Dubè, J. Richer, A. Vinet and R. Gulrajani, A comparison of Monodomain and Bidomain reaction-diffusion models for action potential propagation in the human heart. IEEE Trans. Biomed. Eng. 53 (2006) 2425-2434.

[37] P.-A. Raviart, The use of numerical integration in finite element methods for solving parabolic equations. In Topics in Numerical Analysis, edited by J.J.H. Miller. Academic Press (1973) 233-264.

[38] Z. Qu and A. Garfinkel, An advanced algorithm for solving partial differential equation in cardiac conduction. IEEE Trans. Biomed. Eng. 46 (1999) 1166-1168.

[39] A. Quarteroni and A. Valli, Numerical Approximation of Partial Differential Equations. Springer (1997).

[40] S. Scacchi, A hybrid multilevel Schwarz method for the bidomain model. Comput. Methods Appl. Mech. Eng. 197 (2008) 4051-4061. 
[41] S. Scacchi, A multilevel hybrid Newton-Krylov-Schwarz method for the Bidomain model of electrocardiology. Comput. Methods Appl. Mech. Eng. 200 (2011) 717-725.

[42] S. Scacchi, P. Colli Franzone, L.F. Pavarino and B. Taccardi, Computing cardiac recovery maps from electrograms and monophasic action potentials under heterogeneous and ischemic conditions. Math. Mod. Methods Appl. Sci. 20 (2010) 10891127 .

[43] K.B. Skouibine, N. Trayanova and P. Moore, A numerically efficient model for the simulation of defibrillation in an active bidomain sheet of myocardium. Math. Biosci. 166 (2000) 85-100.

[44] B.F. Smith, P. Bjørstad and W.D. Gropp, Domain Decomposition: Parallel Multilevel Methods for Elliptic Partial Differential Equations, Cambridge University Press (1996).

[45] J.A. Southern, G. Plank, E.J. Vigmond and J.P. Whiteley, Solving the coupled system improves computational efficiency of the Bidomain equations. IEEE Trans. Biomed. Eng. 56 (2009) 2404-2412.

[46] J. Sundnes, G.T. Lines, K.A. Mardal and A. Tveito, Multigrid block preconditioning for a coupled system of partial differential equations modeling the electrical activity in the heart. Comput. Methods Biomech. Biomed. Eng. 5 (2002) $397-409$.

[47] J. Sundnes, G.T. Lines and A. Tveito, An operator splitting method for solving the bidomain equations coupled to a volume conductor model for the torso. Math. Biosci. 194 (2005) 233-248.

[48] H. Si, http://tetgen.berlios.de/. Weierstrass Institute for Applied Analysis and Stochastics, Berlin, Germany.

[49] V. Thomée, Galerkin Finite Element Methods for Parabolic Problems. Springer (1997).

[50] A. Toselli and O.B. Widlund, Domain Decomposition Methods: Algorithms and Theory. Comput. Math. Springer-Verlag, Berlin 34 (2004).

[51] J.A. Trangenstein and C. Kim, Operator splitting and adaptive mesh refinement for the Luo-Rudy I model. J. Comput. Phys. 196 (2004) 645-679.

[52] E.J. Vigmond, F. Aguel and N.A. Trayanova, Computational techniques for solving the bidomain equations in three dimensions. IEEE Trans. Biomed. Eng. 49 (2002) 1260-1269.

[53] E.J. Vigmond, R. Weber dos Santos, A.J. Prassl, M. Deo and G. Plank, Solvers for the cardiac bidomain equations. Progr. Biophys. Molec. Biol. 96 (2008) 3-18.

[54] R. Weber dos Santos, G. Plank, S. Bauer and E.J. Vigmond, Parallel multigrid preconditioner for the cardiac bidomain model. IEEE Trans. Biomed. Eng. 51 (2004) 1960-1968.

[55] J.P. Whiteley, An efficient numerical technique for the solution of the monodomain and bidomain equations. IEEE Trans. Biomed. Eng. 53 (2006) 2139-2147.

[56] M. Zaniboni, 3D current-voltage-time surfaces unveil critical repolarization differences underlying similar cardiac action potentials: A model study. Math. Biosci. 233 (2011) 98-110.

[57] X. Zhang, Multilevel Schwarz methods. Numer. Math. 63 (1992) 521-539. 